\title{
DEVELOPMENT OF A GROUND BASED ATOMIC OXYGEN AND VACUUM ULTRAVIOLET RADIATION SIMULATION APPARATUS
}

\author{
A Thesis \\ presented to \\ the Faculty of California Polytechnic State University, \\ San Luis Obispo
}

\author{
In Partial Fulfillment \\ of the Requirements for the Degree \\ Master of Science in Aerospace Engineering
}

by

Max Jay Glicklin

June 2012 
(C) 2012

Max Jay Glicklin

ALL RIGHTS RESERVED 


\title{
COMMITTEE MEMBERSHIP
}

TITLE:

DEVELOPMENT OF A GROUND BASED ATOMIC OXYGEN AND VACUUM ULTRAVIOLET

RADIATION SIMULATION APPARATUS

\author{
AUTHOR: $\quad$ Max Jay Glicklin \\ DATE SUBMITTED: June 2012 \\ COMMITTEE CHAIR: $\quad$ Kira Jorgensen Abercromby, Ph.D.
}

COMMITTEE MEMBER: $\quad$ Sharon K. Miller, Ph.D.

COMMITTEE MEMBER: Tina Jameson, Ph.D.

COMMITTEE MEMBER: $\quad$ Eltahry Elghandour, Ph.D. 


\title{
ABSTRACT \\ DEVELOPMENT OF A GROUND BASED ATOMIC OXYGEN AND VACUUM ULTRAVIOLET RADIATION SIMULATION APPARATUS
}

\author{
Max Jay Glicklin
}

The space environment possesses numerous unique and unusual attributes, creating challenges that must be considered in order to accomplish a successful space mission. Two of the detrimental aspects of the space environment include Atomic Oxygen, AO, and Ultraviolet, UV, radiation. UV radiation becomes more severe in space as there is no atmosphere to attenuate incoming photons, thereby exposing spacecraft to radiation that never reaches the surface of the Earth. Overall, space vehicles are exposed to a total of 107.4 Watts $/ \mathrm{m}^{2}$ of light shorter than $400 \mathrm{~nm}$. AO is created by the photo disassociation of molecular oxygen by UV radiation with wavelengths less than $\sim 242.1$ $\mathrm{nm}$. AO is a major portion of the neutral atmosphere, and is the dominant species for altitudes between 180 and $675 \mathrm{~km}$. Each of these environments can cause significant damage to spacecraft materials as they have sufficient energy to break molecular bonds: a generalization of AO energy is $4.5+/-1 \mathrm{eV}$ while Vacuum Ultraviolet, VUV, radiation can break bonds as strong as $12.4 \mathrm{eV}$. Synergistic affects are observed when these two environments interact with materials simultaneously, resulting in an accelerated erosion rate. An apparatus has been developed in California Polytechnic State University's, Cal Poly's, space environments laboratory that can simulate the AO and VUV environments individually and simultaneously. This apparatus utilizes a radio frequency, RF, generator to produce a capacitively coupled plasma to create AO in conjunction with a deuterium lamp capable of emitting UV radiation as short as $115 \mathrm{~nm}$. The system has been shown to produce an AO flux of $1.70+/-0.07 \cdot 10^{16}$ atoms $/ \mathrm{cm}^{2}$ while providing an equivalent sun power 4.5 times greater the solar output in the 120-200 nm region of UV light; all of this has been performed at a base pressure near 175 mTorr. Long duration tests of 24 hours, which would be analogous to durations used in a material interaction study, have shown an effective fluence of $1.47+/-0.06 \cdot 10^{21}$ atoms $/ \mathrm{cm}^{2}$, which would equate to an orbital exposure on the order of weeks to months. For the same duration a sample can be exposed to 108 equivalent sun hours of $120-200 \mathrm{~nm}$ radiation. Results from the simultaneous exposure also manifested an accelerated erosion rate, the expected synergetic reactions between the two environments. 


\section{ACKNOWLEDGMENTS}

The author would like to thank Dr. Kira Abercromby of California Polytechnic State University for acting as the advisor for this thesis. Also, Sharon K. Miller of NASA Glenn Research Center who provided invaluable insight and technical knowledge in order to successfully design and operate the system. Thanks to NASA Johnson Space Center for loaning the spectrometer used to perform analyses, as well as Sheldahl Brand materials for their donations of spacecraft materials. Finally, the project sponsors, NASA Jet Propulsion Laboratory and Lockheed Martin, whose funding made this all possible. 


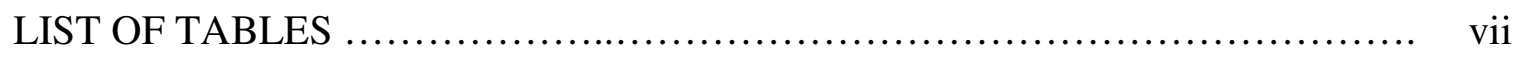

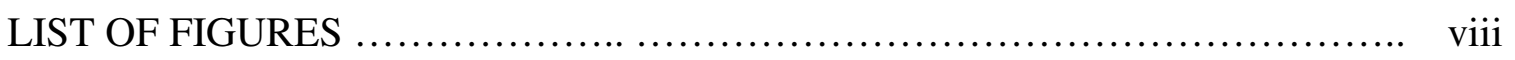

\section{CHAPTER}

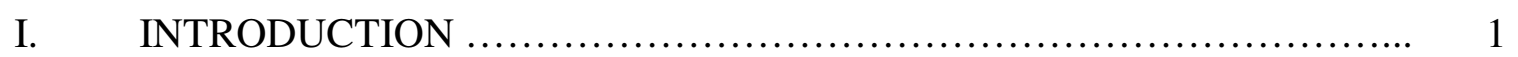

II. MATERIAL INTERACTION WITH ATOMIC OXYGEN AND

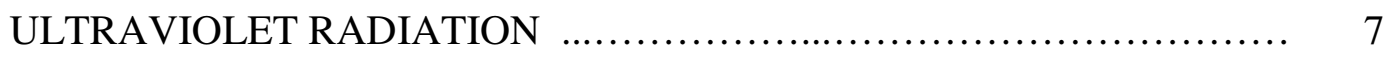

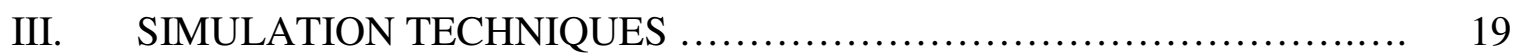

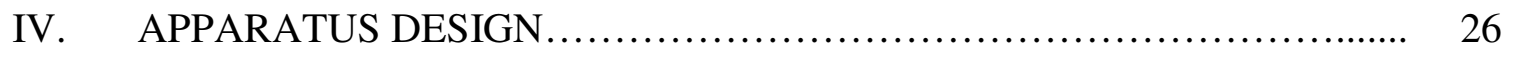

V. SYSTEM CALIBRATION ..................................... 38

VI. RESULTS AND VERIFICATION ................................ 44

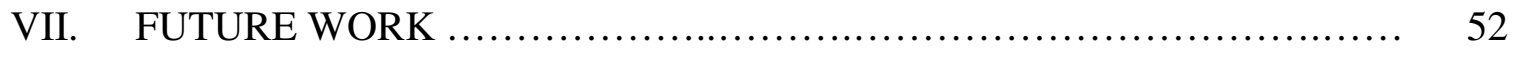

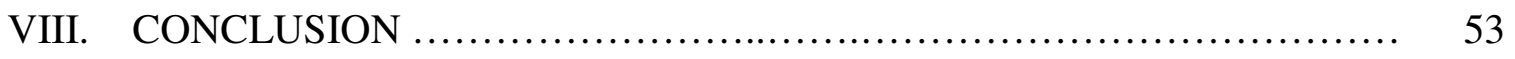

REFERENCES ................................................... 56

\section{APPENDICES}

A. Vacuum Chamber Operation Procedures .......................... 59

B. Atomic Oxygen Apparatus Operation Procedures ................... 60

C. Vacuum Ultraviolet Lamp Operation Procedures .................... 67

D. Lessons Learned .......................................... 68 


\section{LIST OF TABLES}

Table

Page

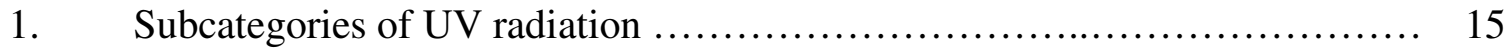

2. Strength of chemical bonds in relation to wavelength $\ldots \ldots \ldots \ldots \ldots \ldots \ldots \ldots \ldots \ldots \ldots$

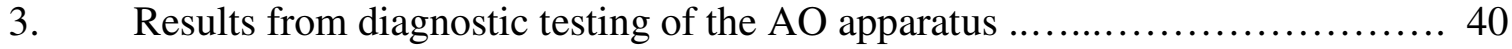

4. Results from long duration $\mathrm{AO}$ and VUV exposure $\ldots \ldots \ldots \ldots \ldots \ldots \ldots \ldots \ldots \ldots$ 


\section{LIST OF FIGURES}

Figure

1. Number density of ambient gases with respect to altitude based on the NRLMSISE-00 model 8

2. Variation of AO number density due to solar output based on the

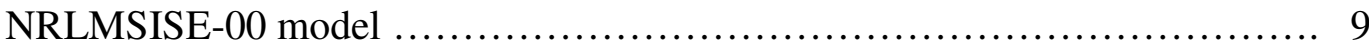

3. Atmospheric temperature versus altitude based upon the

NRLMSISE-00 model 10

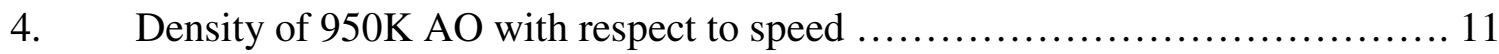

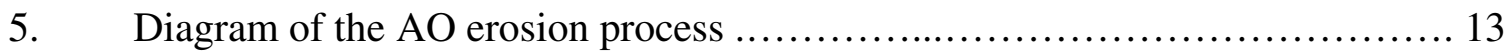

6. AM0 solar irradiance for wavelengths less than $400 \mathrm{~nm} \ldots \ldots \ldots \ldots \ldots \ldots \ldots \ldots$

7. General schematic of a simple capacitively coupled plasma system ........... 27

8. Photograph of the Seren IPS R301MKII RF generator ..................... 29

9. Photograph of the Seren IPS AT3 matching network and the MC2 controller ... 29

10. Cross section of the assembled AO apparatus .............................. 32

11. Exploded view of the upper portion of the AO apparatus .................... 33

12. Exploded view of the lower portion of the AO apparatus ...................... 34 
13. Photograph of the Hamamatsu L10706 Deuterium lamp and power supply ...... 35

14. Manufacturer's expected spectral distribution of the L10706 deuterium

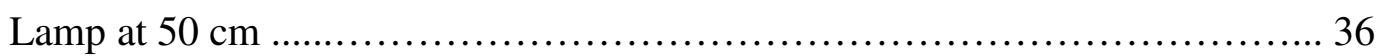

15. Photograph of the chamber with AO apparatus and VUV light source installed; bell jar not included ...................................... 37

16. Equivalent Sun power of the L10706 deuterium lamp based on sample

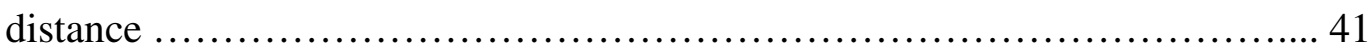

17. Spectral irradiance for AM0 solar output and the L10706 operating at 1

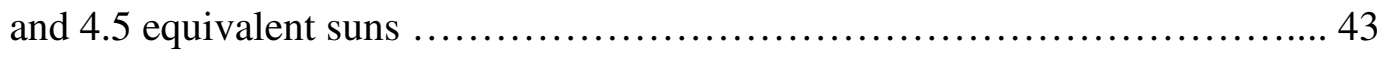

18. Photograph of the AO plasma operating at the long duration test

configuration 44

19. Photograph of a Kapton HN sample subjected to a 24 hour exposure .......... 48

20. Spectral comparison between nominal and eroded Kapton HN ............... 49

21. Spectral output of the AO plasma while operating on $~ 92 \%$ oxygen ............ 50 


\section{Introduction}

The space environment has a unique set of characteristics that create significant challenges for the design and operation of a spacecraft. These features can drive the design of a vehicle or mission, and if they are ignored will seriously compromise mission success and severely inhibit the operational lifetime of a spacecraft. Being in a stable orbit means there are effectively no acceleration gravitational forces, which creates difficulties in handling fluids and operating mechanical mechanisms. Certain aspects are simply created by the lack of atmosphere: vehicles must withstand the vacuum of space, which induces reactions such as outgassing and substantial pressure differentials. Without an atmosphere to block solar radiation, vehicles are now exposed to harsh solar winds and intense ultraviolet, UV, radiation. This also eliminates thermal pathways besides radiation, making vehicles a standalone entity requiring specialized thermal control systems.

The numerous interactions between the space environment and spacecraft can be attributed to the ambient environment surrounding Earth, which is based on orbital location, and the environment generated by the spacecraft themselves. The challenges that engineers must consider are highly dependent on the vehicle's orbital location as well as the vehicle's material composition, layout, and function. There are five primary classes of spacecraft orbits, each with their own unique environments and orbital parameters. Low Earth Orbit, LEO, is classified as having an orbital altitude of less than 1000 kilometers and an inclination less than $65^{\circ}$. Medium Earth Orbit, MEO, has similar inclinations but an altitude range greater than 1000 and less than $36000 \mathrm{~km}$, where Geostationary Orbit, GEO, has an altitude of $36000 \mathrm{~km}$ and an inclination around $0^{\circ}$. The 
last of the Earth orbits are the Polar Earth Orbits, PEO, which pass over the Earth's poles. These orbits have an inclination greater than $65^{\circ}$ and can have any range of altitudes. The final classification refers to orbital trajectories outside the Sphere of Influence of the Earth, and are known as Interplanetary Orbits. ${ }^{1}$

In order to better understand and study the space environment, engineers and scientists commonly refer to the regions of space based on the aforementioned orbital families as well as individual classes of the space environment. The classes are defined based on their composition, structure, or state of matter; the primary four categories are commonly referred to as the neutral, plasma, radiation, and particulate environments.

The neutral environment consists of neutral particles which in essence are an extension of the Earth's atmosphere and remains close to the surface of the Earth. Although these neutrals may not have large amounts of energy, in combination with the orbital velocities associated with LEO these particle interactions can have serious effects on a spacecraft. Collisions result in a transfer of momentum between the particles and the vehicles causing spacecraft drag. These drag forces are large enough to deorbit low flying vehicles and make it necessary to perform propulsive maneuvers to boost vehicles back into their desired orbits. ${ }^{2}$ If there is a separation between the vehicle's center of pressure and center of mass these drag forces will cause a torque, and left uncorrected, this will cause the vehicle to rotate. Mechanical degradation can occur when colliding neutrals have enough energy to remove surface molecules in a process known as sputtering. Chemical degradation also occurs when vehicles interact with atomic oxygen, $\mathrm{AO}$, which can break and form chemical bonds. All of the molecules in the neutral environment can condense on or around spacecraft, leading to the alteration of surface material properties 
which may reduce the efficiencies of thermal control surfaces, solar cells, and onboard sensors. A phenomenon known as spacecraft glow occurs when the ambient environment reacts with vehicle contaminates, such as outgassed materials or thruster firings, and can emit electromagnetic radiation ranging from far UV to infrared. ${ }^{1,2}$

Plasma is present across all orbital families; it is cold and dense in the lower Earth orbits and becomes more energetic and sparse with altitude. These charged particles, which can originate from solar flares or from photoionization of the neutral atmosphere, can cause more serious effects in GEO due to its diminished protection from Earth's magnetosphere. The primary adverse affects are related to spacecraft arcing caused by a buildup of ions and electrons known as spacecraft charging. Arcing occurs when these charges redistribute themselves due to a potential difference; which can damage electronics and materials. Arcing can occur in several modes that are dependent on potential differences or discharge energy; these destructive mechanisms include electrostatic discharge, dielectric breakdown, metallization melt, bulk breakdown, surface breakdown, and induced currents. ${ }^{2}$ Charging can also disturb spacecraft operations by causing ground shifts, electromagnetic torques, and interference with communications systems and sensors. ${ }^{1}$

The radiation environment consists of extremely energetic particles such as photons, charge particles, and neutrons which can be divided into three primary regimes. ${ }^{1}$ The Van Allen belts consist of energetic protons and electron trapped in Earth's magnetosphere, forming toroidal belts reaching outwards to over $60000 \mathrm{~km}$ in altitude. Radiation from the sun consists of solar ejections knows as Solar Particle Events, Coronal Mass Ejections, and the Solar Wind, as well as solar irradiance which emits across the 
spectrum from X-rays through the infra-red. Energetic particles which originate from interplanetary sources are known as Galactic Cosmic Rays. The most energetic of these events, the Galactic Cosmic Rays, can produce particles with energies exceeding $10^{19} \mathrm{eV}$, yet have small fluxes on the order of 4 particles $/ \mathrm{cm}^{2} / \mathrm{s}$. ${ }^{2}$ Radiation can penetrate deep into materials causing reactions such as dielectric charging and displacement damage, the creation of interstitial sites in a material. This can have detrimental effects on electronic hardware; incoming particles interact with internal semiconductors causing internal arcing and currents. This leads to electric upsets such as connection burnouts, memory errors caused by bit flips, an increase in signal noise, clock resets, and even damaging the semiconductors in solar cells inhibiting power production. ${ }^{1} \mathrm{UV}$ radiation affects surface materials by breaking bonds and altering the absorptance and emittance of thermal control surfaces. Solar radiation also comprises of radiofrequency electromagnetic waves extending up to $50 \mathrm{MHz},{ }^{1}$ which can interfere with spacecraft communication systems operating at similar frequencies.

The particulate environment consists of natural meteoroids and manmade debris created by spacecraft and launch vehicle operations. In rare occurrences large amounts of debris can be created during intentional or unintentional collisions. Smaller natural particles, less than $1 \mathrm{~cm}$ in diameter, usually have velocities between $15-20 \mathrm{~km} / \mathrm{s}$, but can reach velocities exceeding $70 \mathrm{~km} / \mathrm{s} .{ }^{1}$ These particles contain large amount of kinetic energy, but can be shielded against. Debris greater than $\sim 10 \mathrm{~cm}$ in diameter can be tracked and possibly avoided; the greatest risk is particles ranging between $1-10 \mathrm{~cm}$ which are typically too small to track and too large to shield against. Collisions with orbital debris can damage surfaced and exposed underlying material, or completely destroy hardware 
such as solar cells. Less dangerous debris can be carried to space on the surfaces of the spacecraft itself, creating a nearby debris cloud. In some cases this debris cloud has been shown to interfere with spacecraft sensors such as star trackers. ${ }^{1}$

Many of these environments act synergistically, making it difficult to predict or simulate the affects of the space environment on a spacecraft mission. Radiation can increase the amount of outgassed materials, and once ionized can be attracted back to a vehicles which has been charged by ambient plasma. Collisions with micrometeoroids can leave underlying materials susceptible to plasma charging or erosion by the neutral environment. Charging of a vehicle essentially changes the amount of energy required to sputter surface materials, greatly accelerating the process when collisions with the neutral environment are numerous. ${ }^{2}$ Both atomic oxygen and incoming UV radiation have enough energy to break molecular bonds, and in conjunction increase the erosion rates of materials.

With an endless number of possible interactions scientists and engineers must evaluate and study the effects of the space environment to achieve a successful mission in space. One of the greatest challenges was that spacecraft could not be returned to Earth undamaged, making it incredibly difficult to analyze the space environment. With the advent of the space shuttle, experiments could be performed in-situ and be safely returned for evaluations. Many of the shuttle's first missions carried samples of common materials to evaluation their reactions to the environments. The space shuttle also delivered and retrieved the Long Duration Exposure Facility, LDEF, to and from LEO; this vehicle was coated entirely with experiments meant to study the space environment and provided an enormous amount of flight data. Since then the Materials Internal Space Station 
Experiment, MISSE, has placed several Passive Experiment Containers, PECs, on the International Space Station, ISS, to further develop a database of material interactions. ${ }^{3}$ Collecting flight data is time consuming and expensive, making it difficult to qualify new materials for operation in space. With the growing desire to have vehicles remain in orbit for prolonged durations it has become even more important to perform ground based simulations and models to analyze and predict a material's performance in the space environment.

The goal of this thesis to develop a ground based apparatus that can simulate the effects of the Low Earth Orbit Environment on materials through the reproduction of certain aspects of the neutral and radiation environment. By using a vacuum chamber retrofitted with a plasma generator and a deuterium lamp, the destructive nature of atomic oxygen and vacuum ultraviolet radiation will be studied through independent and simultaneous exposure experiments designed to manifest synergistic results. 


\section{Material Interaction with Atomic Oxygen and Ultraviolet Radiation}

Atomic oxygen is single oxygen atoms created by photo disassociation of diatomic oxygen, $\mathrm{O}_{2}$, by UV radiation. The strength of the double bond in $\mathrm{O}_{2}$ is $5.12 \mathrm{eV} ;{ }^{4}$ the wavelength required to break a bond is calculated using the equation

$$
E=\frac{h * c}{\lambda}
$$

where $\mathrm{E}$ is the bond energy, $h$ is Planck's constant of $4.135 \times 10^{-15} \mathrm{eV} / \mathrm{s}, c$ is the speed of light, and $\lambda$ is the wavelength. Using Eq. (1), one observes that any wavelength of light shorter than $\sim 242.1 \mathrm{~nm}$ will have enough energy to break diatomic oxygen into AO. On orbit, the mean free path $\mathrm{AO}$ atoms is on the order of $10^{8}$ meters, which lowers the probability that the $\mathrm{AO}$ will recombine into ozone, $\mathrm{O}_{3} .{ }^{5}$ Shorter wavelengths, $10-100 \mathrm{~nm}$, are accredited with the photo ionization of $\mathrm{AO}$ and other molecules. ${ }^{1}$

Not only is it important to understand the process in which AO is created, but also where it naturally occurs. Figure 1 shows the number density of the molecules in the neutral atmosphere with respect to altitude. This is figure was generated using the Naval Research Laboratory Mass Spectrometer Incoherent Scatter model from the year 2000, NRLMSISE-00, data built into MathWorks MATLAB's Aerospace Toolbox. ${ }^{6}$ This is an empirical model created by the Naval Research Laboratory that takes data from satellites and high altitude sounding rockets to create an atmospheric model accurate to $1000 \mathrm{~km}{ }^{7}$ As a quick supplemental fact, the $\mathrm{E}$ on the end of the initialism signifies that the model extends from the surface of the Earth into the exosphere. The values in Fig. 1 are based on the yearly predicted average from 2012. The anomalous oxygen seen in Fig. 1 refers high energy oxygen atoms which are either ionized or have high thermal energies. 


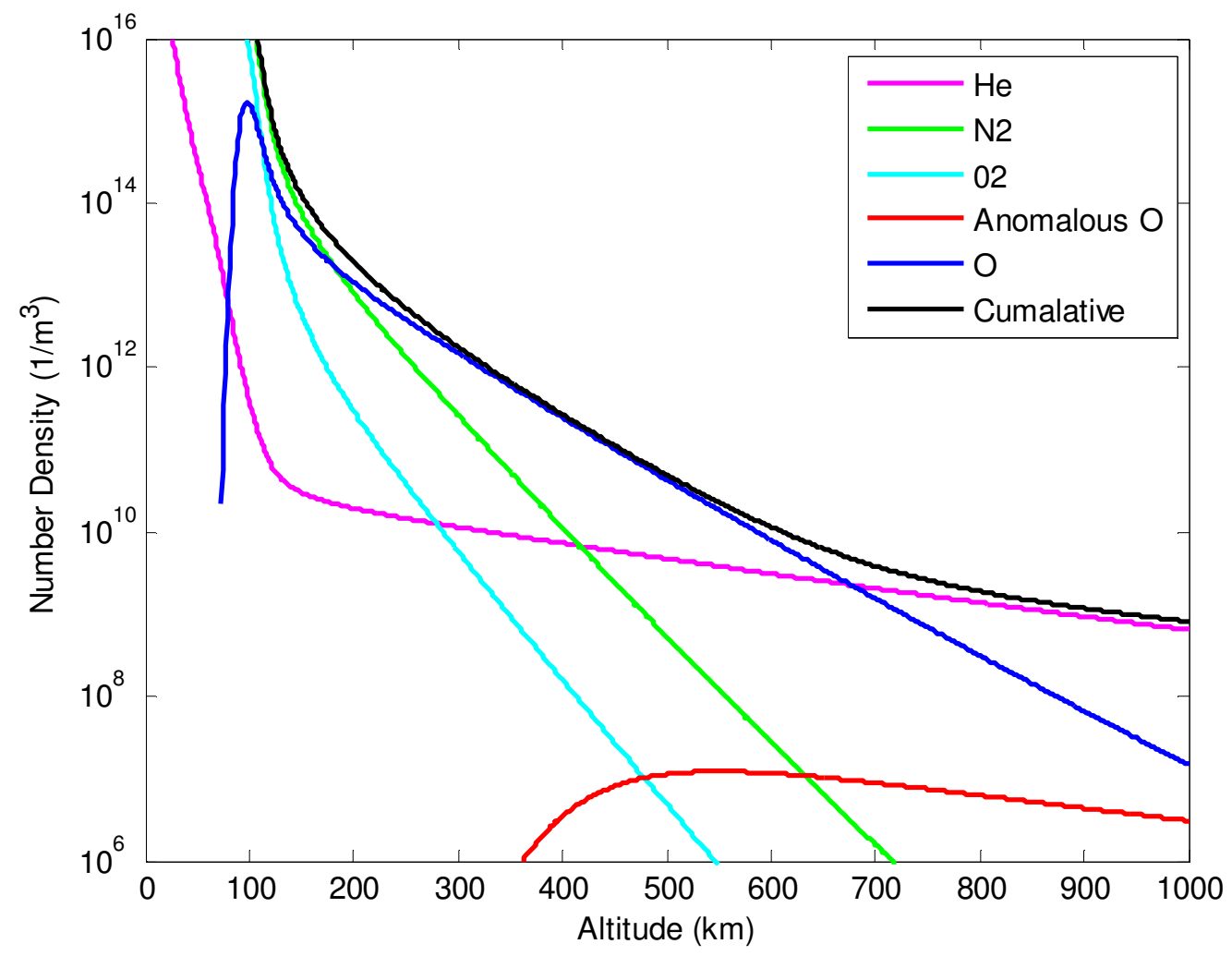

Figure 1: Number density of ambient gases with respect to altitude based on the NRLMSISE-00 model.

Within a single year, the values can vary greatly with solar fluctuations, making it incredibly hard to predict the number density of $\mathrm{AO}$ at a given altiude. ${ }^{5}$ The solar minimums and maximums occur on a 11 year cycle causing much variation in the annual AO density. This changes the AO flux, the rate that particles pass through a given area, measured as atoms $/ \mathrm{cm}^{2} / \mathrm{s}$, witnessed by a spacecraft. An evaluation of a $400 \mathrm{~km}$ circular orbit using the MSIS-86 data showed a 12 year local minimum of AO fluence, or the total number of particles collisions in a defined time, measured as atoms $/ \mathrm{cm}^{2}$, of less than $1.0 \cdot 10^{21}$ atoms $/ \mathrm{cm}^{2}$ in 2006 while a local maximum was predicted to exceed $3.0 \bullet 10^{21}$ atoms $/ \mathrm{cm}^{2}$ in $2013 .^{5}$ 
Figure 2 demonstrates how variations in the solar flux can vary the AO number density in certain locations by a factor of several hundred depending on the current solar output.

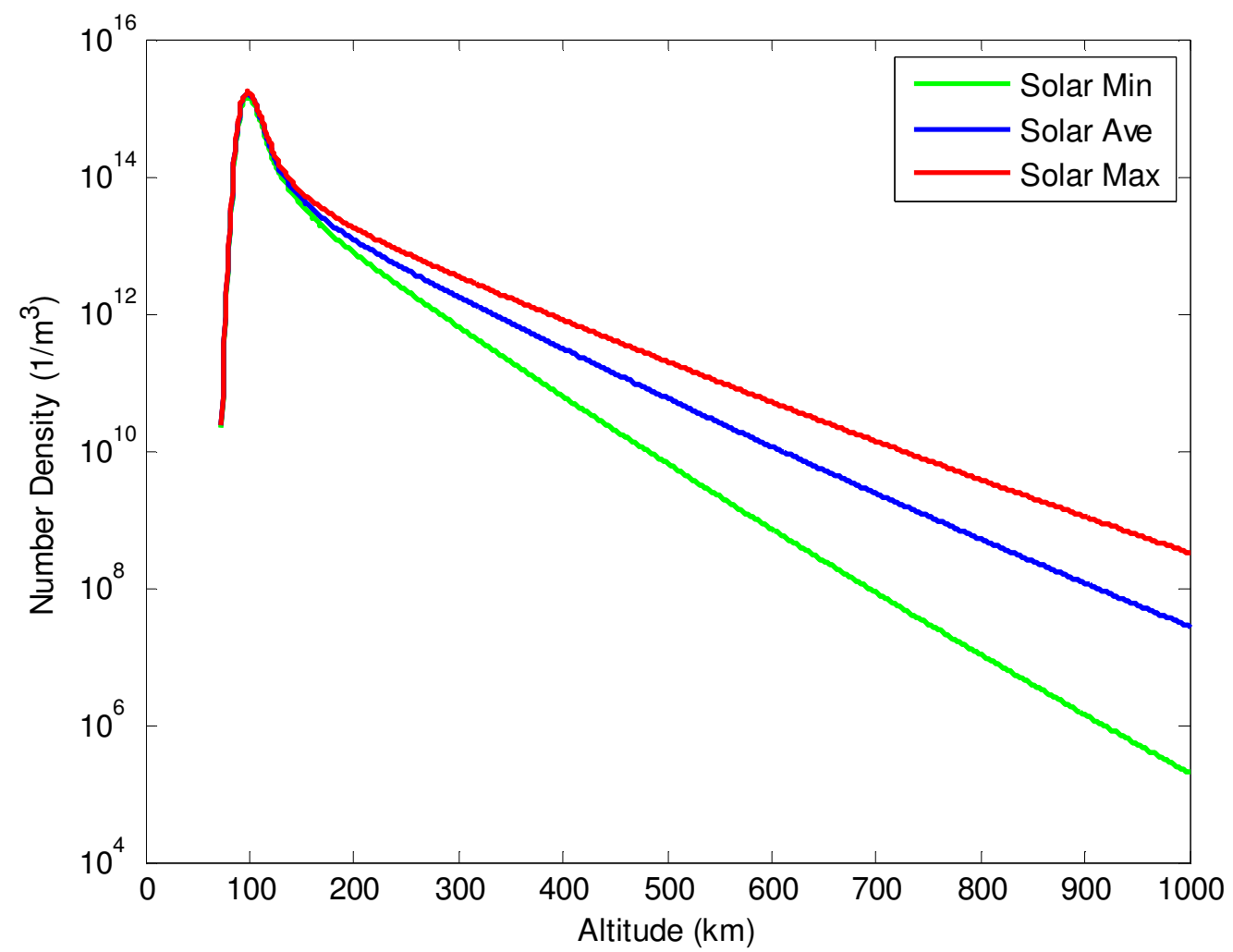

Figure 2. Variation of AO number density due to solar output based on the NRLMSISE-00 model.

From Fig. 1, AO is the dominant species between 180 and $675 \mathrm{~km}$; it is preceded by a diatomic nitrogen environment and superseded by a helium environment. As the number density of $\mathrm{AO}$ begins to drop rapidly with altitude, the effects of $\mathrm{AO}$ are inconsequential at high orbital altitudes.

The ambient AO energy is based upon the thermal energy of the thermosphere and exosphere. The temperature of Earth's atmosphere was calculated using the NRLMSISE-00 model and can be seen in Fig. 3. The model shows an average exospheric temperature of $~ 950$ Kelvin. Converting into electron volts using Boltzmann's constant 
of $8.617 \times 10^{-5} \mathrm{eV} / \mathrm{K}$ gives an approximate ambient energy of $0.08 \mathrm{eV}$. However, the fact that orbital speeds in LEO range between 7 and $8 \mathrm{~km} / \mathrm{s}$ drastically increase the collision energy between $\mathrm{AO}$ and a spacecraft. Using the equation for kinetic energy,

$$
E=\frac{1}{2} * m * v^{2}
$$

for a vehicle with an orbital speed of $7.5 \mathrm{~km} / \mathrm{s}$ (equivalent to an orbital altitude of $715 \mathrm{~km}$ ) along with the mass of a single $\mathrm{AO}$ atom of $16 \mathrm{amu}$ results in an energy of $4.66 \mathrm{eV}$. Because of orbital motion almost all AO erosion occurs in the ram direction, the face of the spacecraft normal to the vehicle's velocity vector.

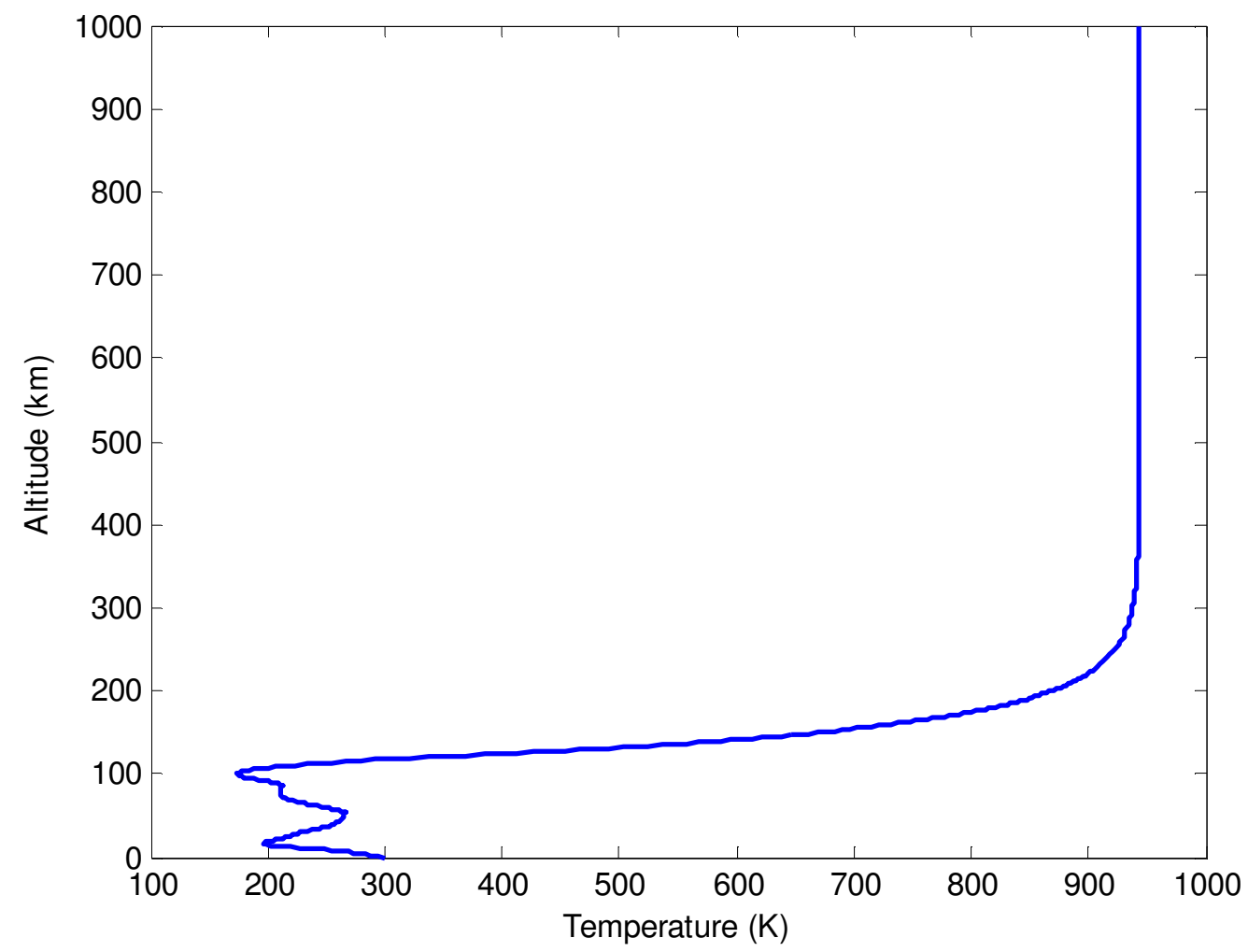

Figure 3: Atmospheric temperature versus altitude based upon the NRLMSISE-00 model.

The kinetic theory of gases asserts that certain properties of $\mathrm{AO}$ can be described using a Maxwellian distribution; a density function of $950 \mathrm{~K} \mathrm{AO}$ with respect to its 
speed can be seen in Fig. 4. This means that a small number of AO atoms can approach the vehicle from the anti-ram direction and interact with the surface materials. Since the collision energy levels of $\mathrm{AO}$ are dependent on numerous factors such as the orbital parameters, the co-rotation of Earth's atmosphere, and the thermal velocity of the atoms themselves, ${ }^{5}$ it is difficult to assign a singular energy value to AO. To establish familiarity with AO energy levels, an assessment of a stereotypical Low Earth Orbit with an eccentricity of 0 , an inclination of $28.5^{\circ}$, an altitude of $400 \mathrm{~km}$, and an average thermosphere temperature of $1000 \mathrm{~K}$ would have typical AO energies of $4.5+/-1 \mathrm{eV} .^{5}$

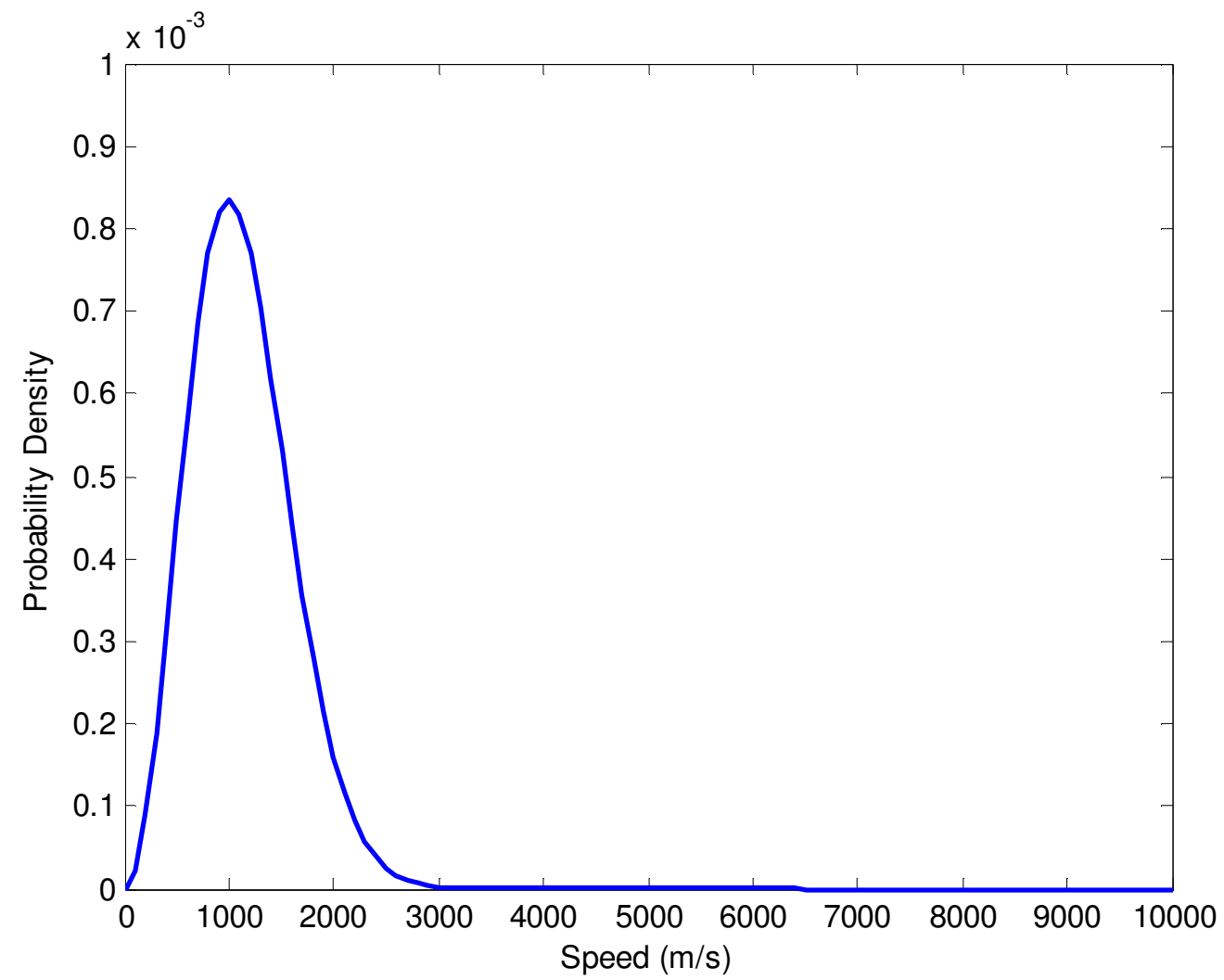

Figure 4: Probability Density of 950K AO with respect to speed.

$\mathrm{AO}$ is corrosive because the collision energy is enough to break bonds causing the erosion of surface materials via the loss of oxidations products. ${ }^{5}$ The reaction efficiency of $\mathrm{AO}$ erosion is measured in the volumetric loss per incident oxygen atom, $\mathrm{cm}^{3} /$ atom, 
and is used to compare erosion rates of different materials. There tends to be three types of reactions between materials and AO. The first are materials that form oxides, and includes most organic materials, the second are materials that retard further oxidation, and the third are materials that form volatile oxides at a continuous rate. ${ }^{8}$

Polymeric materials, such hydrocarbons and fluorocarbons, typically experience the highest reaction efficiencies; ${ }^{9,10}$ as materials in this family are commonly used in space for their functional properties, precautions need to be taken for any vehicle operating in an $\mathrm{AO}$ environment. There are numerous erosion mechanisms which can occur on unprotected surfaces. Pits and cones form on the surface of the material causing surface texturing and thinning. This texting changes the appearance of the material by increasing the material's diffuse reflectance while decreasing its specular transmittance, ${ }^{5}$ which will also alter the thermal absorptance and emittance of said material. AO will oxidize surface layers, and can form either volatile or non-volatile oxides. The erosion mechanism becomes more intricate when evaluating multi-layer insulation or materials. Each layer will have its own response and reaction efficiency, and therefore erode at different rates. If a successive layer with a higher reaction efficiency becomes exposed, it will erode away material beneath an existing cover layer in a process known as undercutting.

It is also important to realize that because most LEO spacecraft have inclination greater than $0^{\circ}$ there is an angular component associated with $\mathrm{AO}$ erosion as the vehicles have latitudinal oscillations around the equator. This modifies the corrosive mechanism as it performs a sweeping motion across the body in question. The consequence of this sweeping action reduces the prominence of the cones that form on the surface materials. ${ }^{11}$ 
An example of the chemical erosion process of $\mathrm{AO}$ on a hypothetical graphite surface can be seen in Fig. 5. The first reaction consists of an $\mathrm{AO}$ atom colliding with the graphite surface, breaking the $7.4 \mathrm{eV}$ carbon-carbon double and forming a surface oxide. The new surface molecule has a bond strength of $13.1 \mathrm{eV}$, and with a subsequent $\mathrm{AO}$ collision with an energy greater than $1.7 \mathrm{eV}$ will release the surface molecule in the form of a carbon monoxide molecule with a triple bond. This carbon monoxide bond has a strength of $11.4 \mathrm{eV}$, completely the energy well balance. ${ }^{1}$ A diagram of the above process can be seen in Fig. 5.

I. Chemical Bonding

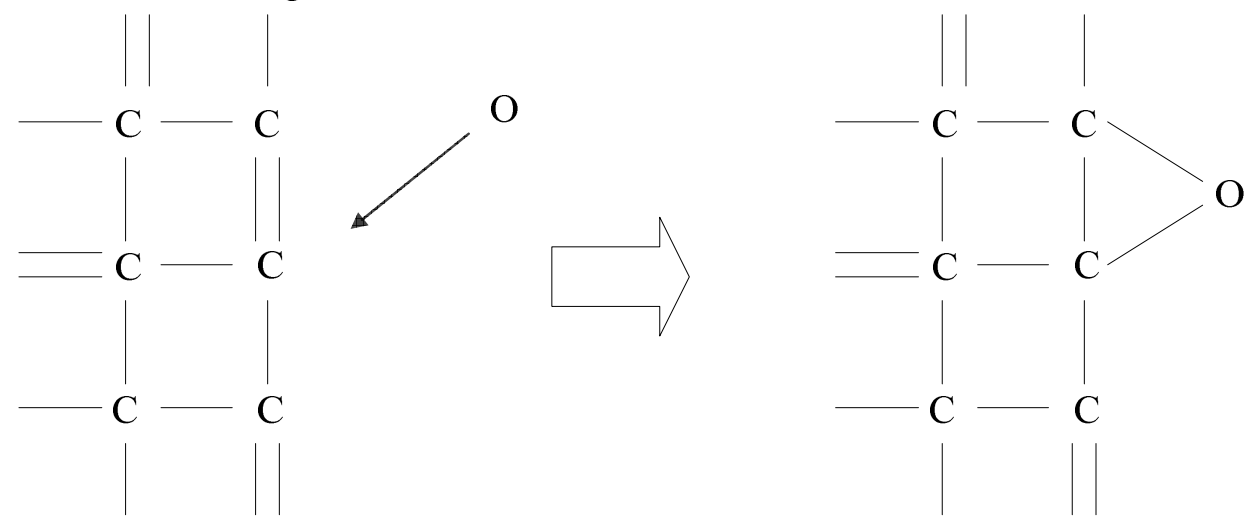

II. Physical Removal

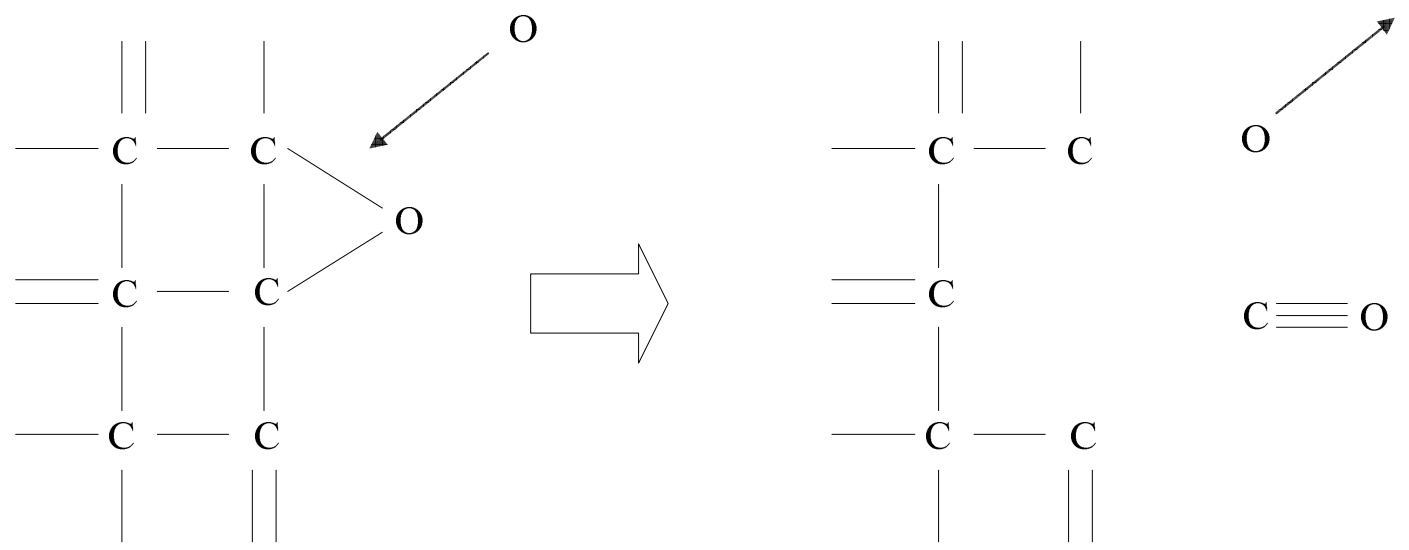

Figure 5: Diagram of the AO erosion process. 
The AO flux seen by a spacecraft is highly dependent on the vehicles orbital parameters such as altitude, eccentricity, inclination, etc. ${ }^{5}$ Preventative measures must be taken to mitigate the effects of $\mathrm{AO}$ for any spacecraft that will be continuously exposed to the AO environment. One practical method approach is simply coating the external surfaces, especially the surfaces that will be on the ram facing side of the spacecraft, with a coating that will form non-volatile oxides. Silicon coatings are rather effective as they form a stable oxide and protect underlying materials. ${ }^{5,12}$ Most metals also form stable surface oxides to produce the same effect. There are numerous materials that can be selected, ranging from compounds like $\mathrm{SiO}_{2}$ and $\mathrm{Al}_{2} \mathrm{O}_{3}$ to pure metals like germanium, aluminum, and gold. In each case only a thin surface layer on the order of $100 \mathrm{~nm}$ is required to provide adequate protection. ${ }^{2,5}$ Impurities such as atoms or molecules present inside of material can also arrest $\mathrm{AO}$ erosion, if a material with this trait was optically evaluated after a directional $\mathrm{AO}$ attack it would reveal conic structures with the arresting particle on top. This is because in a directional attack the molecule, usually a stable oxide, would protect the underlying material.

Each material requires specific evaluation before use in an $\mathrm{AO}$ environment. For instance, silver oxides do not adhere to their parent surfaces. This continuous spalling has been accredited for the failure of solar cell interconnects that are made of silver. ${ }^{1}$ The materials designed to protect materials can also introduce new, unforeseen reactions. For instance, silicones can lead to surface cracking and flaking, ${ }^{13}$ which may cause harm to vehicle operations. Any thin protective coating has the potential to be scratched or cracked during material handling during vehicle integration. This can cause the exposure of underneath layers and allow for undercutting erosion. ${ }^{11}$ Even the roughness of the 
polymer surface or the configuration of the protective coating can act as failure mode. ${ }^{11}$

Coatings that unknowingly cease to provide effective protection can potentially generate anomalous behavior with indeterminate root causes. A solution to this problem is the use of non-carbon based polymers such as siloxanes. The reaction efficiency of siloxane can be as much at two orders of magnitude less than organic polymers, ${ }^{13}$ reducing the need for special surface coatings.

Ultraviolet radiation is divided into subcategories based upon wavelength and photon energies. On Earth these categories include UV-A, UV-B, and UV-C; and are the primary groups for rating protective sunscreens and sunglasses. Radiation below $290 \mathrm{~nm}$ is absorbed in the atmosphere, and is responsible for creating the ozone layer which resides between an altitude of $10-50 \mathrm{~km} .{ }^{4}$ At orbital altitudes, spacecraft and their surface materials are exposed to unfiltered UV radiation and a new set of subcategories have been defined. For convenience, these subcategories have not only been divided by wavelength, but have also been converted to express photon energies in $\mathrm{eV}$ with the use of Eq. (1). The wavelength groupings can be seen in Table 1.

Table 1. Subcategories of UV radiation. ${ }^{4}$

\begin{tabular}{|c|c|c|c|}
\hline Name & Initialism & Wavelength, nm & Energy, eV \\
\hline Near ultraviolet & NUV & $400-300$ & $3.1-4.1$ \\
\hline Middle ultraviolet & MUV & $300-200$ & $4.1-6.2$ \\
\hline Vacuum ultraviolet & VUV & $200-100$ & $6.2-12.4$ \\
\hline Extreme ultraviolet & EUV & $100-10$ & $12.4-124$ \\
\hline
\end{tabular}

The shortest primary emission from the sun is the Lyman-alpha line of hydrogen, which emits at $121 \mathrm{~nm} .{ }^{14}$ A standard has been produced by the American Society for Testing and Materials, ASTM, which accurately defines the solar constant for zero-airmass, AM0, meaning the data represents the solar output without any atmospheric attenuation. ${ }^{15}$ The data for this standard, ASTM E-0490, has been developed through the 
use of high altitude aircraft and spacecraft with the appropriate measurements devices as well as Earth based observations and solar models. Figure 6 was produced with data from this standard, and shows the solar irradiance for all wavelengths less than 400nm.

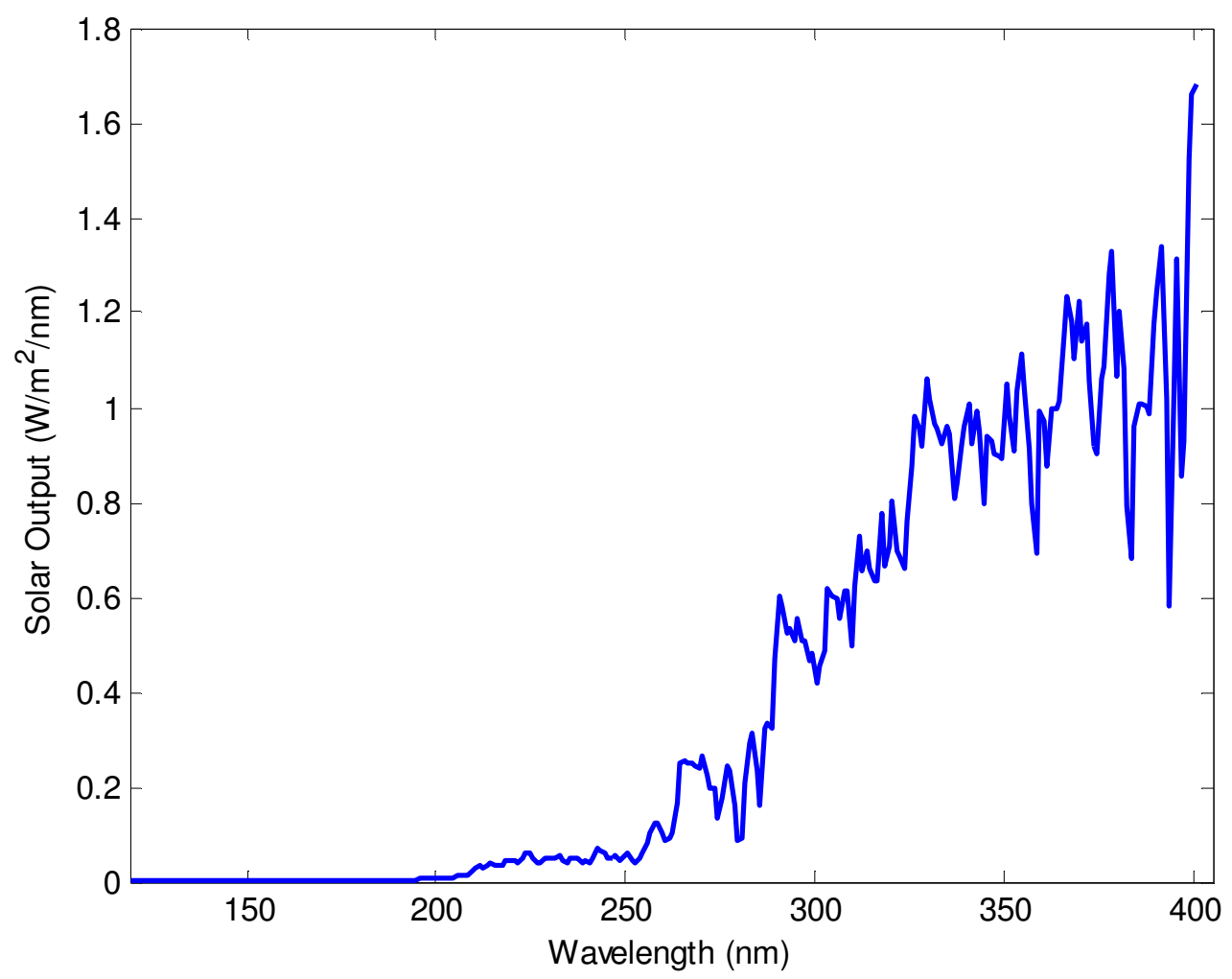

Figure 6. AM0 solar irradiance for wavelengths less than $400 \mathrm{~nm} .{ }^{15}$

Although this portion of the spectrum only accounts for $7.86 \%$ of the entire solar constant, ${ }^{15}$ this regime can have considerable effects on spacecraft. UV radiation is absorbed in the surface layers of materials, and cause photochemical reactions. These reactions can change optical, mechanical, and chemical properties of the incident material. ${ }^{14}$ Optical changes can have a significant effect on spacecraft operations as any change in the thermal properties of a material, such as absorptance and emittance, can disrupt the vehicle's thermal balance. Although UV radiation has a very shallow penetration depth, the photons have enough energy to break many organic bonds. ${ }^{14,16}$ 
Table 2 shows a number of common atomic bonds that are present in polymers used on spacecraft. If you notice the bond strengths do not match those presented in Fig. 5, this is because the bonds in Fig. 5 are part of a larger polymeric chain in which the nearby covalent bonds increase the apparent bond strength.

Table 2. Strength of chemical bonds in relation to wavelength. ${ }^{2}$

\begin{tabular}{|c|c|c|c|}
\hline Chemical & Bond Type & $\begin{array}{c}\text { Bond Energy } \\
\left(@ 25^{\circ} \mathrm{C}\right)(\mathrm{eV})\end{array}$ & $\begin{array}{c}\text { Wavelength } \\
(\mathrm{nm})\end{array}$ \\
\hline C-C & Single & 3.47 & 360 \\
\hline C-N & Single & 3.17 & 390 \\
\hline C-O & Single & 3.73 & 330 \\
\hline C-S & Single & 2.82 & 440 \\
\hline N-N & Single & 1.69 & 730 \\
\hline O-O & Single & 1.52 & 820 \\
\hline Si-Si & Single & 2.30 & 540 \\
\hline S-S & Single & 2.52 & 490 \\
\hline C-C & Double & 6.29 & 200 \\
\hline C-N & Double & 6.38 & 190 \\
\hline C-O & Double & 7.64 & 160 \\
\hline C-C & Triple & 8.59 & 140 \\
\hline C-N & Triple & 9.24 & 130 \\
\hline C-O & Triple & 7.77 & 160 \\
\hline
\end{tabular}

As shown above, incoming radiation will have enough energy to break the molecular bonds in polymers; shorter wavelengths mean more energy, and an increased probability that the incoming photon will sever a bond. Physically what is happening is that as bonds break the molecules in a material become smaller, in essence this introduces dislocations in the material resulting in a harder and more brittle material.

AO and UV radiation work synergistically, as they can both have sufficient energy to break molecular bonds and trigger chemical reactions, in conjunction they can lead to the accelerated erosion of a material depending on the types of bonds present in the material itself. 
These complex interactions manifest the need to better understand material interactions with the LEO environment. Without adequate analysis, a spacecraft mission can be jeopardized if the materials and expected orbital environment are not considered. On orbit testing provides valuable empirical information, but cannot be relied upon due to cost and time constraints. In order to qualify new materials for spaceflight, or to perform accelerated exposure to predict long term effects, ground based analysis must be performed. 


\section{Simulation Techniques}

As there are numerous applications that require access to LEO, it becomes important to establish ground based studies and simulations to better understand the environment and its effects on spacecraft. This statement holds even more value when considering the monetary investment necessary to place a vehicle on orbit. In an ideal world, an apparatus would exist that could simulate all aspects of the space environment and exactly replicate every aspect of the spacecraft's predicted environment. As the need to evaluate spacecraft and environmental interactions still exists, methods have been developed to generate computer models and simulation chambers to predict or evaluate these interactions.

The first phase in simulating the LEO environments begins with AO and UV, as these environments incite considerable effects on material properties. Computational models can predict the erosion of materials, ${ }^{10,17}$ but cannot provide the same amount of reassurance as physical results. Placing the materials in question on orbit for collection of empirical data is possible and has been done on numerous occasions, but it is costly, time consuming, and cannot provide accelerated testing for long term durability predictions. The solution is ground based testing in a system that can simulate the desired environments. This is the preferred method as it can provide valuable information for a relatively inexpensive cost while allowing the operator to easily modify environmental parameters to correlate with the environment in question.

Interest in $\mathrm{AO}$ erosion increased after the interactions were observed on returning shuttle missions. Numerous methods of ground based simulation were developed as part of NASA's Atomic Oxygen Effects Programs. ${ }^{8}$ Systems have been developed that can 
produces $\mathrm{AO}$ energies from $0.1 \mathrm{eV}$ isotropic thermal plasmas to $70 \mathrm{eV}$ hyperthermal directed plasmas. ${ }^{11}$ The most common methods use either Radio Frequency, RF, microwave, or laser energy in order to disassociate diatomic oxygen into AO.

Direction and energy play an important role in the erosion mechanism. Low energy levels $\mathrm{AO}$ plasmas are typically isotropic, meaning the AO is scattered, traveling at low speeds in all directions. Erosion from this type of plasma will cause surface roughening, leading to a more diffuse response, but will not form the tall pits and cones associated with directional orbital AO. The low energy thermal plasma can be manipulated through interactions with geometric surfaces or magnetics to form a directional discharge; this will cause the pits and cones to grow larger, but still will not compare to the erosion of materials on the ram facing side of a spacecraft. ${ }^{18}$ Low energy RF plasmas and microwave discharges can still provide pertinent results, recalling the Maxwellian distribution from Chapter II, a certain amount of these low energy particles will have enough energy to replicate $\mathrm{AO}$ erosion. ${ }^{18}$ Every incident $\mathrm{AO}$ atom that interacts with a surface material has a finite probability of reacting with said material, this probability decreases as the overall energy of the plasma decreases. In order to compensate for this loss, low energy plasma systems must produce a higher number density of AO to manifest the desired results.

Energetic AO ions have a spectral line that emits UV radiation at a wavelength of $130 \mathrm{~nm},{ }^{18-20}$ which can accelerate the erosion process as discussed in Chapter II. Methods have been developed that can eliminate a direct line of sight between the AO source and the samples in order to reduce this side affect. ${ }^{19,21}$ 
Further considerations include thermal heating and charging. The AO reaction efficiency of materials typically increases with temperature, meaning an elevated temperature will result in greater than expected erosion. This can simply be accommodated for by cooling the samples, such as a water cooled apparatus. ${ }^{8}$ Plasma systems also have to take into account that ion will rapidly charge samples, causing further ions to be repelled, reducing the effective flux. ${ }^{8}$ Also, high energy sources on the order of tens of $\mathrm{eV}$ must consider the required ion energy to begin sputtering any surrounding materials. If incoming ions have enough energy to sputter surrounding materials, surface molecules could be removed and lead to sample contamination, altering the results of the study. ${ }^{19}$ Each individual apparatus and its operational parameters must be evaluated in order to mitigate these contamination risks.

Currently, the best methods for creating $\mathrm{AO}$ in ground based facilities includes but are not limited to plasma ashers, which utilize either inductively or capacitively coupled plasmas, continuous or pulsed lasers, gridded or gridless ion sources, or microwave electron cyclotron resonance sources.

Plasma ashers use RF energy to create thermal energy plasma around $0.1 \mathrm{eV} .^{8,12}$ Typically these systems are utilized as a capacitively coupled plasma, CCP, or an inductively coupled plasma, ICP. RF energy ranging from $0.1-100 \mathrm{MHz}$ can be used, but the standard frequency is $13.56 \mathrm{MHz}$, a frequency band set aside by the Federal Communications Commission, FCC, for industrial plasma applications. ${ }^{22}$ These systems tend to be the most practical systems in terms of cost and simplicity, as well as their ability to be scaled to provide large area and high flux exposures. ${ }^{18}$ One version at NASA's Glenn Research Center in Cleveland, Ohio, has an exposure area of 1.5 by 2.1 
m. ${ }^{23}$ These systems will run either on air or oxygen at pressures below 200 mTorr while provided fluxes on the order of $10^{15}$ atoms $/ \mathrm{cm}^{2} / \mathrm{s}$ or greater. ${ }^{18,22}$ When operating on air it has been found the ambient nitrogen, which also becomes ionized, does not affect the results of $\mathrm{AO}$ erosion. ${ }^{18}$

As mentioned before, although plasma ashers create an AO plasma that is characteristically different from orbital AO they still generate material erosion that is qualitatively similar to orbital AO. ${ }^{12}$ To reiterate, because the plasma is isotropic, meaning the velocities of the plasma are omnidirectional, the $\mathrm{AO}$ erosion in a plasma asher will not exhibit the same cones formed in a high energy directional plasma. This still causes surface roughing and optical alterations, but no conic structures. ${ }^{18}$ Based upon the reaction probabilities, these thermal energy plasma require a considerably higher flux to produce the same level of oxidation. ${ }^{18}$ Considerations must also be made when evaluating polymers with chlorine and fluorine structures, as these bonds will experience anomalous erosion yields greater than what would occur on orbit. ${ }^{11}$

Electron Cyclotron Resonance, ECR, is a plasma source which operates by gyrating electrons at the same phase of an induced magnetic field. ${ }^{22}$ This provides sufficient energy to disassociate diatomic oxygen via energetic electron collisions, ${ }^{21}$ and have shown to operate at medium vacuum pressures on the order of $10^{-4}$ Torr. An ECR system used at Glenn Research Center is highly adjustable and can produce a directed $13 \mathrm{eV}$ AO beam or a scattered isotropic beam $\sim 0.04 \mathrm{eV},{ }^{18,21}$ with the abilities to provide neutral AO between $0.04-0.1 \mathrm{eV} .{ }^{19}$ In addition, this facility has an unique arrangement that can block the majority of the $130 \mathrm{~nm}$ light emitted by the plasma from contacting the sample area. 
Ion beam sources have also been used in the form of gridded sources and gridless sources; gridless sources are also known as End Hall sources. These sources typically operate on pure oxygen on has a discharge made primarily of $\mathrm{O}_{2}{ }^{+}$, with negligible $\mathrm{O}^{+}$and $\mathrm{O}^{++} .{ }^{11}$ These systems are capable of producing very high energies up to $\sim 70 \mathrm{eV}$, but are limited in the overall flux which can be emitted. ${ }^{11,23}$ Numerous methods exist to neutralize the ion beams, such as interactions with solid surfaces, a gas phase charge exchange, or through photo-detachment. ${ }^{23}$

The last method that will be discussed in this thesis employs a laser to induce the breakdown of molecular oxygen. These systems typically use a $\mathrm{CO}_{2}$ which is designed to either be pulsed or supply a continuous wave. The laser is directed into a nozzle which has been filled with diatomic oxygen while providing enough thermal heat to create a blast wave. This causes the atomic oxygen to form on the blast wave that is directed at the sample area. The nozzle design can be customized to provide specific exit velocities while also neutralizing the beam through ion-electron interactions. These systems show an overall performance similar to LEO, as it can operate at medium vacuum pressures, supply a mono-energetic $\mathrm{AO}$ beam around $5 \mathrm{eV}$, and provide a high flux for accelerated exposure. $^{23}$

In order to maintain comparable and reliable data across multiple AO erosion facilities, ASTM E2089 was established. ${ }^{24}$ The most recent version was reapproved in 2006 and covers topics such as standard operating procedures and methods to quantify the effective flux and fluence of an $\mathrm{AO}$ apparatus. The standard establishes four wellunderstood witness materials that are recommended for use in determine AO flux and fluence; these materials are Kapton polyimide (H or HN), TFE fluorocarbon fluorinated 
ethylene propylene (FEP), low density polyethylene, and pyrolytic graphite. Advisory methods with regard to sample preparation, handling, sizing, and masking/cladding are outlined. A standard is also defined for proper vacuum dehydration and weighing of material; samples should be outgassed for a minimum of 48 hours at a pressure below 200 mTorr and be weighed within 5 minutes of removal of the vacuum in order to reduce uncertainties with respect to the reabsorption of vapors. The two methods for determining effective flux and fluence consist of mass loss calculations and thickness loss calculations, these calculations are dependent on an assumed in-space erosion yield (reaction efficiency). ${ }^{24}$

In order to simulate the UV radiation environment special lamps are required. As mentioned in Chapter II, the shorter wavelengths are absorbed by the atmosphere, requiring the lamps to be operated under vacuum. Most transparent materials also block short wavelengths, which requires the lamps to have special windows. In order to achieve wavelengths in the VUV spectrum a deuterium (a hydrogen isotope) lamp must be used in conjunction with a Magnesium Fluoride, $\mathrm{MgF}_{2}$, window which allows for wavelengths as short as $115 \mathrm{~nm}$ to be transmitted. These lamps also require special Cesium-Iodide phototubes for calibration. ${ }^{19}$ For the NUV and MUV regimes there is a greater variety of lamp and windows choices. In typical space simulators either xenon or xenon-mercury short arc lamps are used with a fused silica window. ${ }^{12}$ One important consideration is restricting the exposure intensity. Solar exposure is typically described in equivalent suns, G, and equivalent sun hours, ESH. Common practice is to limit the numbers of suns to between 3 and 5, as this range of exposure is not expected to change the erosion mechanism. ${ }^{12}$ An important consideration must be made when using UV lamps to 
simulate solar radiation; no lamp exactly mimics the peaks and troughs of the Sun's spectrum. For instance, deuterium lamps typically have a spectral peak near $160 \mathrm{~nm}$, which is not present in the solar spectrum. If material absorbs radiation at this wavelength, it will experience erosion at a faster rate in the simulated environment than the orbital environment. 


\section{Apparatus}

It was decided to construct a capacitively coupled plasma system for the LEO simulation apparatus to simulate the $\mathrm{AO}$ environments in the space environments laboratory at California Polytechnic State University, Cal Poly. This method was chosen because it is cost effective and relatively simple to construct. A mentioned in the previous chapter, the disadvantages of this system are that $\mathrm{AO}$ will be an isotropic thermal plasma. This means that the AO will have an energy between $\sim 0.04-0.1 \mathrm{eV}$, much lower than the orbital $\mathrm{AO}$ energy of $\sim 4.5 \mathrm{eV}$, and that the $\mathrm{AO}$ will have omnidirectional velocities, as opposed to the directional orbital AO. It will be important to consider these characteristics when performing material studies; the resulting erosion will not form the tall cones and pits as seen on orbital erosion. This will also lead to atypical undercutting when studying materials with protective coatings, assuming surface defects exist. However, this simulation method still provides a qualitatively similar erosion mechanism, and therefore can still provide valuable insight into the effects of $\mathrm{AO}$ on spacecraft materials. The primary advantage of this method is that $\mathrm{CCP}$ can produce very high AO fluxes, allowing for accelerated exposure and testing of materials.

In its simplest form, a CCP is created through the use of two parallel electrodes: one that is grounded and one that is powered. The plasma is generated between the electrodes, and is known as an electrodeless discharge due to a plasma sheath which is

created around the electrodes. ${ }^{22}$ In most applications, the substrate is placed directly inbetween the plate; either in the plasma itself or in the sheath surrounding the ground plate. 
A general schematic of a CCP system can be seen in Fig. 7 Many modifications can be made to the system based on user needs. Common modifications include the addition of devices to control and contain the plasma. An enclosure referred to as a darkspace shield can be used to surround the RF electrode. A narrow gap between the two components that is less than the thickness of the plasma sheath will prevent plasma from forming on the backside of the electrode and minimize secondary emissions. CCP systems also exist that operate on dual RF frequencies, one frequency for each electrode, or one RF powered electrode and one DC biased electrode; these systems are used when modification of the plasma sheath parameters is necessary. ${ }^{22}$

Additional operation modes allow for the electrodes to be removed from the plasma system, such as a plasma generated in an isolated glass chamber with the electrodes mounting to the outside of the glass container. Water cooling of the electrodes is also common when requirements on substrate temperatures exist. ${ }^{22}$

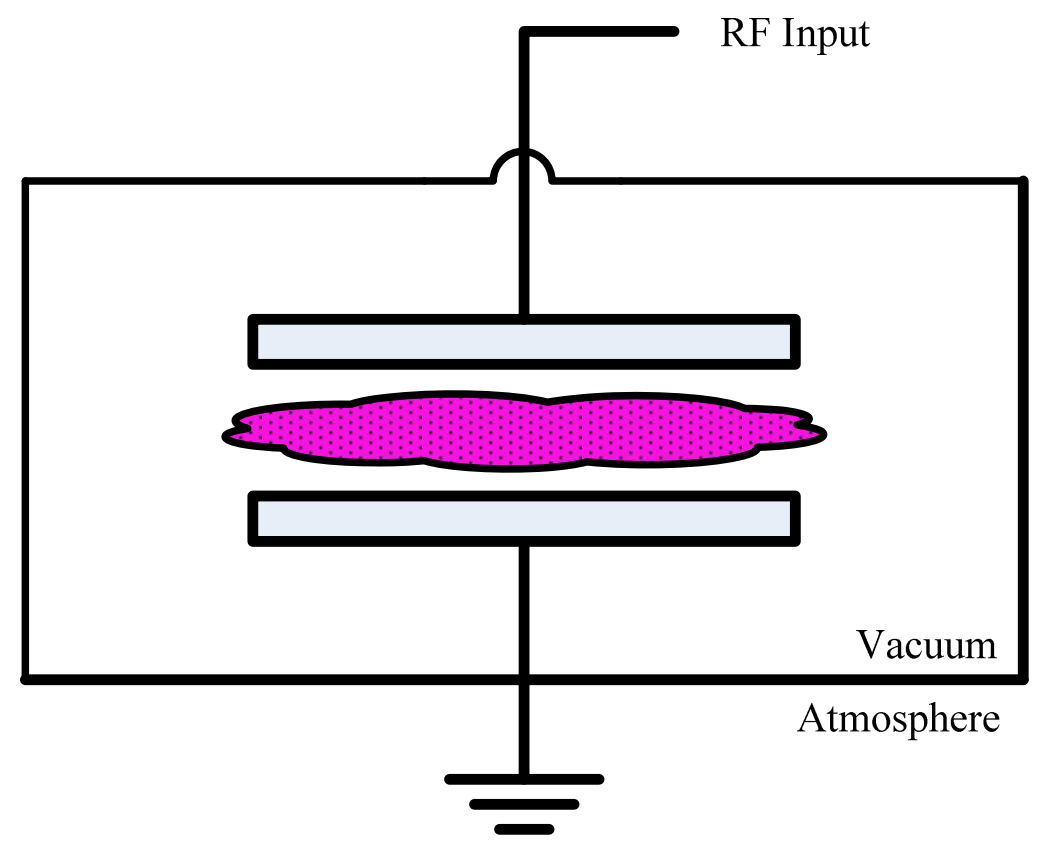

Figure 7. General schematic of a simple capacitively coupled plasma system. 
In order to power the CCP, a comprehensive power supply system manufactured by Seren Industrial Power Systems was used; this package includes a RF generator, load matching network, and system controller. The generator is a Seren R301MKII that operates at a fixed frequency of $13.56 \mathrm{MHz}$ and has a maximum power output of $300 \mathrm{~W}$. This is coupled with an AT3 matching network designed to match the impedance load of the plasma generator; in this case, the load was predetermined by an industry standard of $50 \Omega$. This device is designed to eliminate any reflected signals produced in the load (cables, electrical connections, plasma) which allows the system to forward the maximum power to the plasma while protecting the RF generator from internal damage. The matching network has what is referred to as an L type circuit topology, meaning one capacitor and one inductor to match the impedance of the load. The AT3 accomplishes this task with a load capacitor constructed of three fixed capacitors and one primary variable capacitor which is coupled with a tune mechanism constructed of a smaller variable capacitor and a seven turn, two inch diameter, silver inductor. The system is controlled through user inputs and a Seren MC2 controller which adjusts the variable capacitors in the AT3 matchbox to automatically find the matching impedance. A picture of the RF power system can be seen in Fig. 8 and 9. 


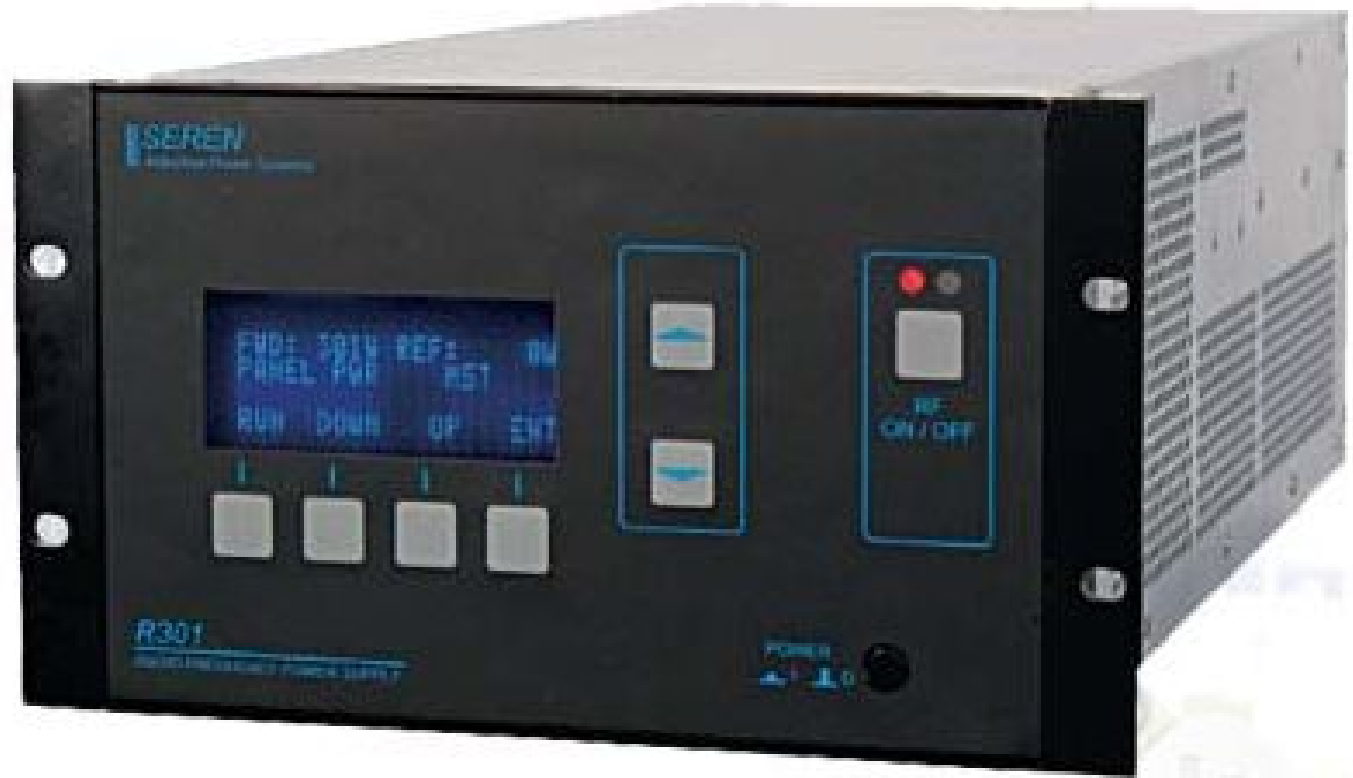

Figure 8. Photograph of the Seren IPS R301MKII RF generator. ${ }^{25}$
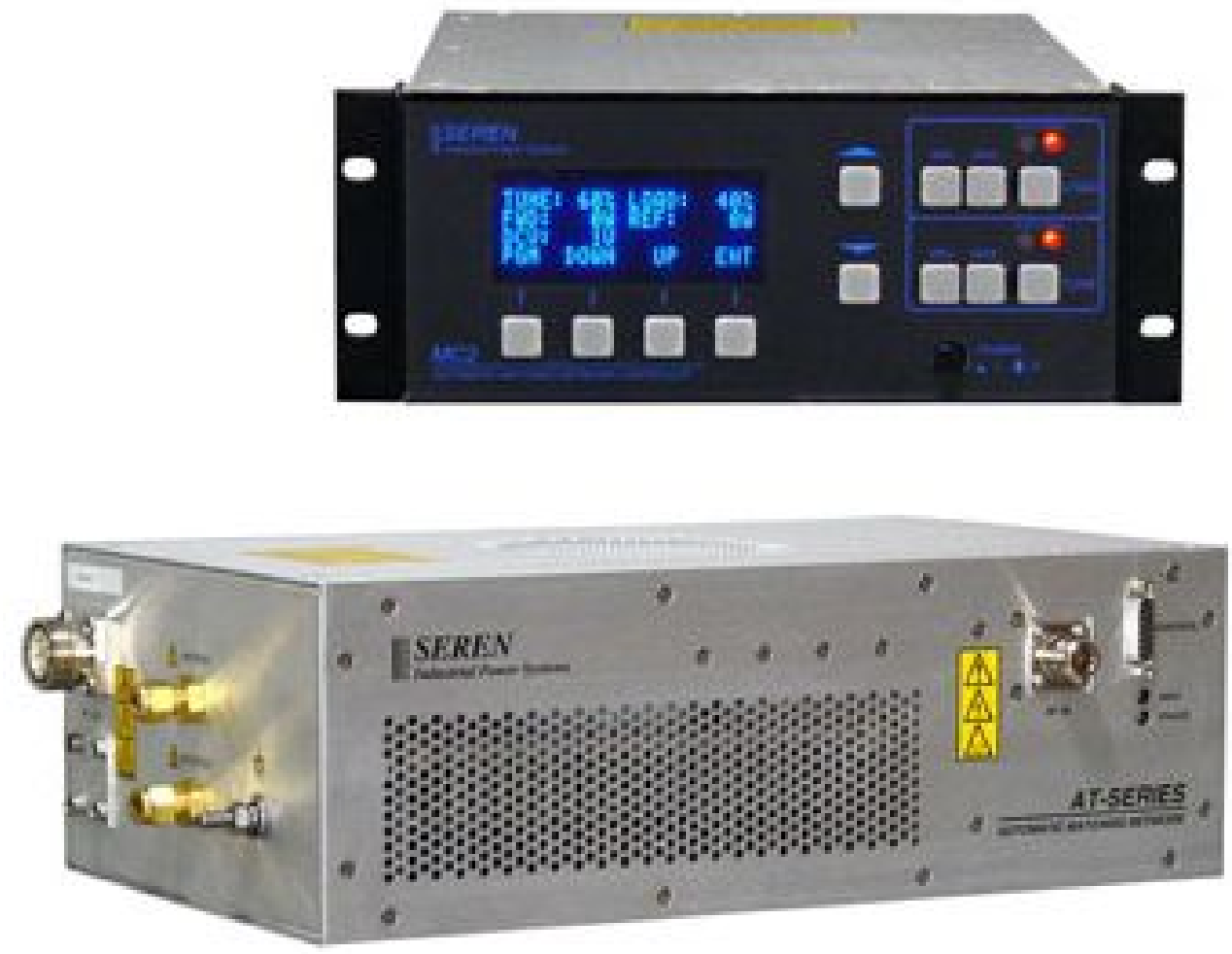

Figure 9. Photograph of the Seren IPS AT3 matching network and the MC2 controller. $^{25}$ 
The AO and UV simulation apparatus was constructed in one of Cal Poly's high vacuum chambers. The chamber is a retrofitted Veeco Model 747 deposition chamber which has been modified for space simulation. The primary chamber consists of a pyrex cylinder approximately $50 \mathrm{~cm}$ in diameter and $32 \mathrm{~cm}$ tall. The chamber has two pumps: the first is a Welch Model 1397 mechanical pump with a pumping speed of 500 liters/min used as the roughing pump, and can achieve a base pressure less than 10 mTorr. The second is a Brooks Automation CTI Cryotorr 10 cryopump. When in use, the chamber can reach a base pressure on the order of $10^{-8}$ Torr. This pump will not be used in this application for safety reasons, but is available for assorted experimentation. Procedures for operating the chamber can be found in Appendix A.

With a goal of producing the highest possible AO flux with a CCP device while having an exposure area large enough to perform materials studies and analysis, the AO apparatus was sized based upon the largest possible RF electrode that could safely and reasonably fit inside of the bell jar.

The RF electrode is a $15.25 \mathrm{~cm}$ aluminum disc that is $0.9 \mathrm{~cm}$ thick; 6061 aluminum alloy was selected due to its relatively high sputtering threshold which will reduce the amount of contamination that may occur. ${ }^{2}$ The electrode has four mounting holes for 1/4"-20 alumina screws which not only provide electrical and thermal isolation, but also assist in alignment of the electrode with the ground plate. A simple blind hole is used for an interference fit (friction fit) to the RF power connector; this connection is robust yet removable.

A dark space shield, DSS, which is designed to minimize the secondary emissions from the electrode to improve the concentration of the $\mathrm{AO}$ the desired region, 
encompasses the RF electrode. The gap distance between the electrode and DSS is approximately $1.9 \mathrm{~mm}$; this value was determined through empirical observations during preliminary apparatus testing that consisted of a free standing RF electrode and a simple plasma shield. This gap distance was selected to eliminate any plasma generation between the electrode and the DSS.

The grounding plate is a $25.4 \mathrm{~cm}$ square aluminum plate mounting on an adjustable stand that allows for variation of the gap distance from 0 to $12 \mathrm{~cm}$. A counter bored through hole in the center of the plate is used to insert the operating gas, air, in between the ground plate and the RF electrode. An aluminum cover plate with a \#8 mirror finished attaches to the ground plate. This plate has four evenly spaced holes used for sample containment; each hole has an identical radial and axial displacements from the RF electrode as recommend by Ref. 18. These holes were precision machined to accurately and consistently control the witness and specimen samples control area. The holes are $2.540+/-0.003 \mathrm{~cm}$ in diameter, outlining and sample exposure area of $5.06 \mathrm{+} /$ $0.02 \mathrm{~cm}^{2}$. There are eight low profile screws that are evenly spaced around each sample area opening to apply even pressure and assure adequate masking of the samples.

The apparatus has been modeled in SolidWorks, ${ }^{26}$ and is seen in Figs. 10,11,12. The upper portion of the apparatus includes the RF electrode, the dark space shield, the RF coaxial power cable, ceramic spacers, and mounting hardware. The lower portion contains the ground plate, gas insertion line, and sample containment plate. The mounting hardware for the lower apparatus is not shown. The dark space shield and ground plate both have $5.08 \mathrm{~cm}$ wide grounding straps mode of Type 101 ultra conductive copper 
alloy; neither of the ground straps are shown. A cross section view of the AO assembly can be seen in Fig. 10.
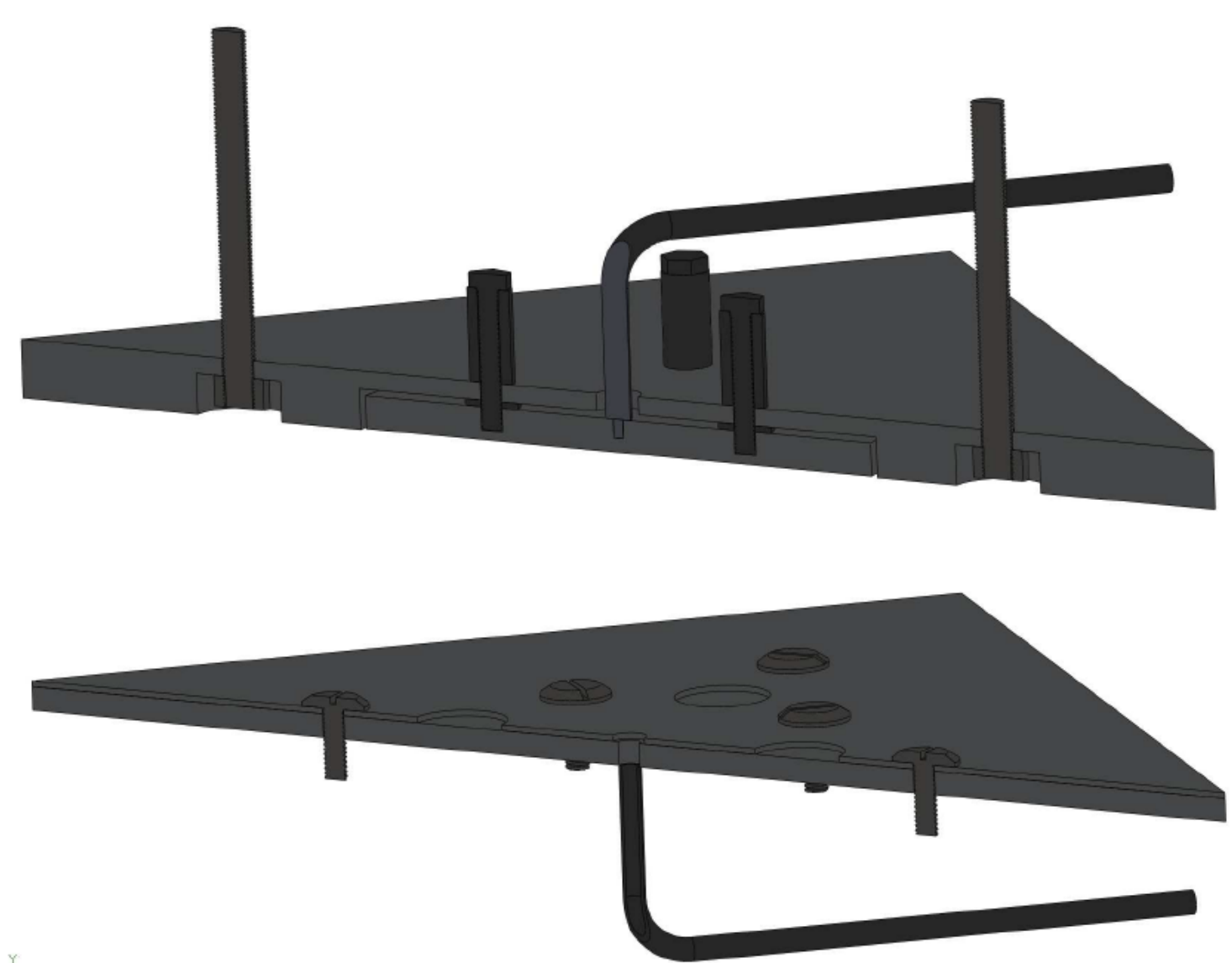

Figure 10. Cross section of the assembled AO apparatus. 


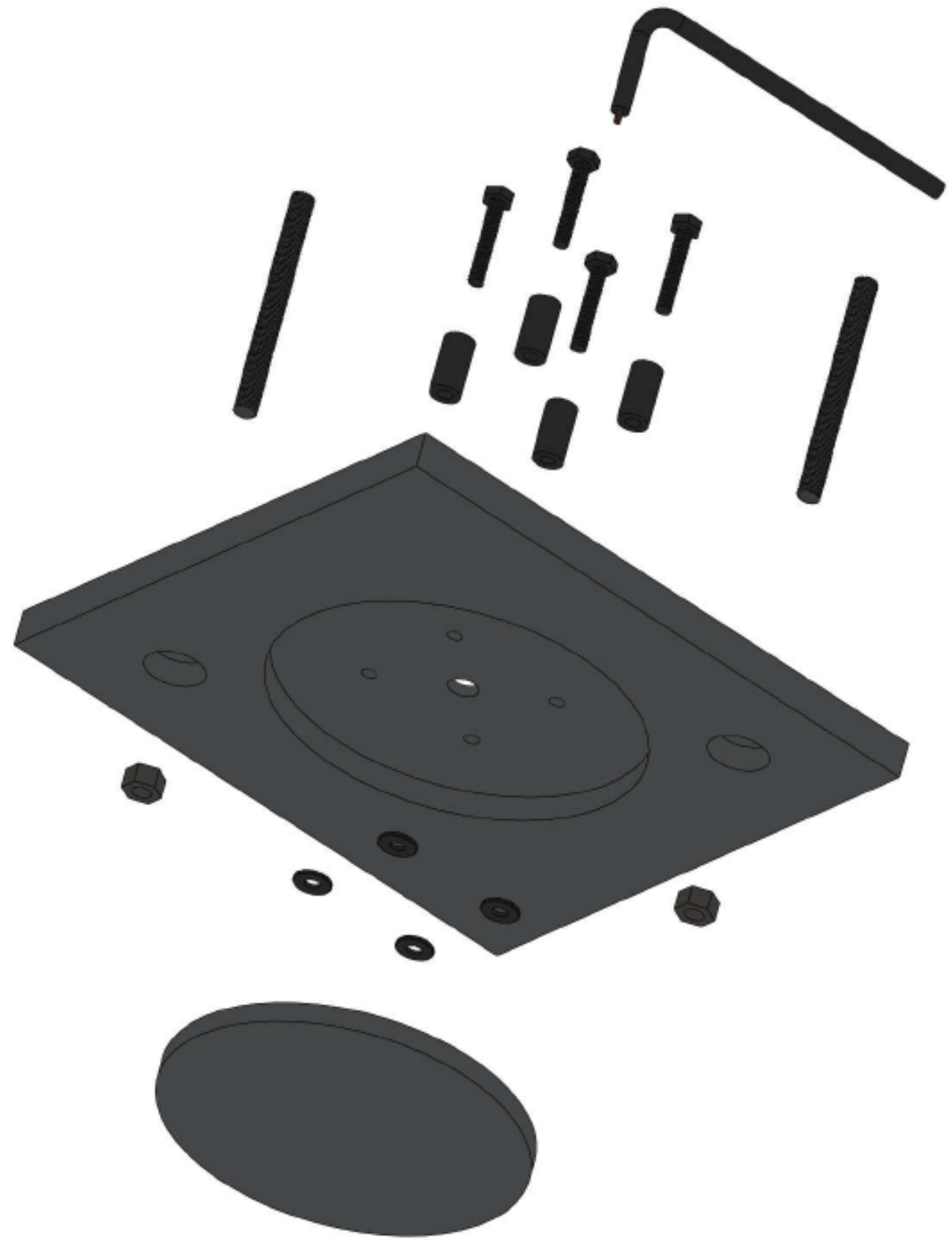

Figure 11. Exploded view of the upper portion of the AO apparatus. 


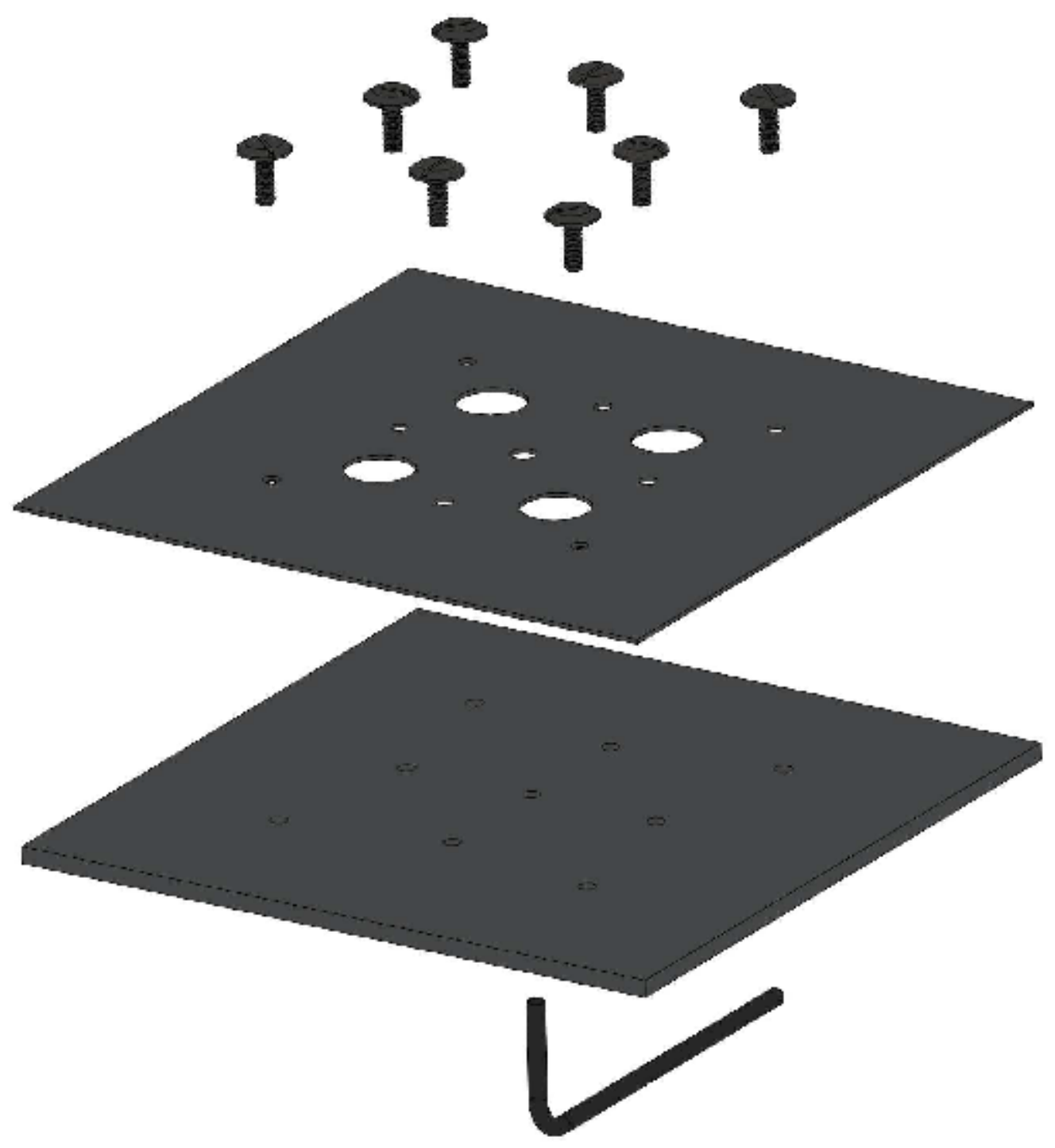

Figure 12. Exploded view of the lower portion of the AO apparatus.

To provide VUV radiation, a deuterium lamp by Hamamatsu Corporation was selected. In order to provided the desired wavelengths of $115 \mathrm{~nm}$ and greater, a lamp with a Magnesium Fluoride window had to be selected. It was also important to confirm that the lamp could provide an equivalent sun power of 3-5 $\mathrm{G}$ in order to provide the maximum possible accelerated exposure without distorting results. Due to limitations on internal space and vacuum feedthrough configurations, the particular lamp selected was the Hamamatsu L10706-500. This lamp is unique in the sense that it mounted at the tip of 
a $50 \mathrm{~cm}$ semi-rigid bellows adjustable arm, and mounted to the chamber on a ConFlat 275 flange. This arm allows for the positioning of the lamp at the desired angle and distance from the target sample; however, this design limits the lamp in size and power, therefore utilizing the least intense lamp manufactured by Hamamatsu. This requires the lamp to be placed relatively close to the sample, and will be discussed in Chapter IV. As the $50 \mathrm{~cm}$ arm is fairly long in comparison to the chamber dimension, the lamp assembly was mounted on top of a ConFlat flange full nipple that is approximately $15 \mathrm{~cm}$ long to essentially reduced the arm length inside of the chamber. A photograph of the lamp can be seen in Fig. 13.

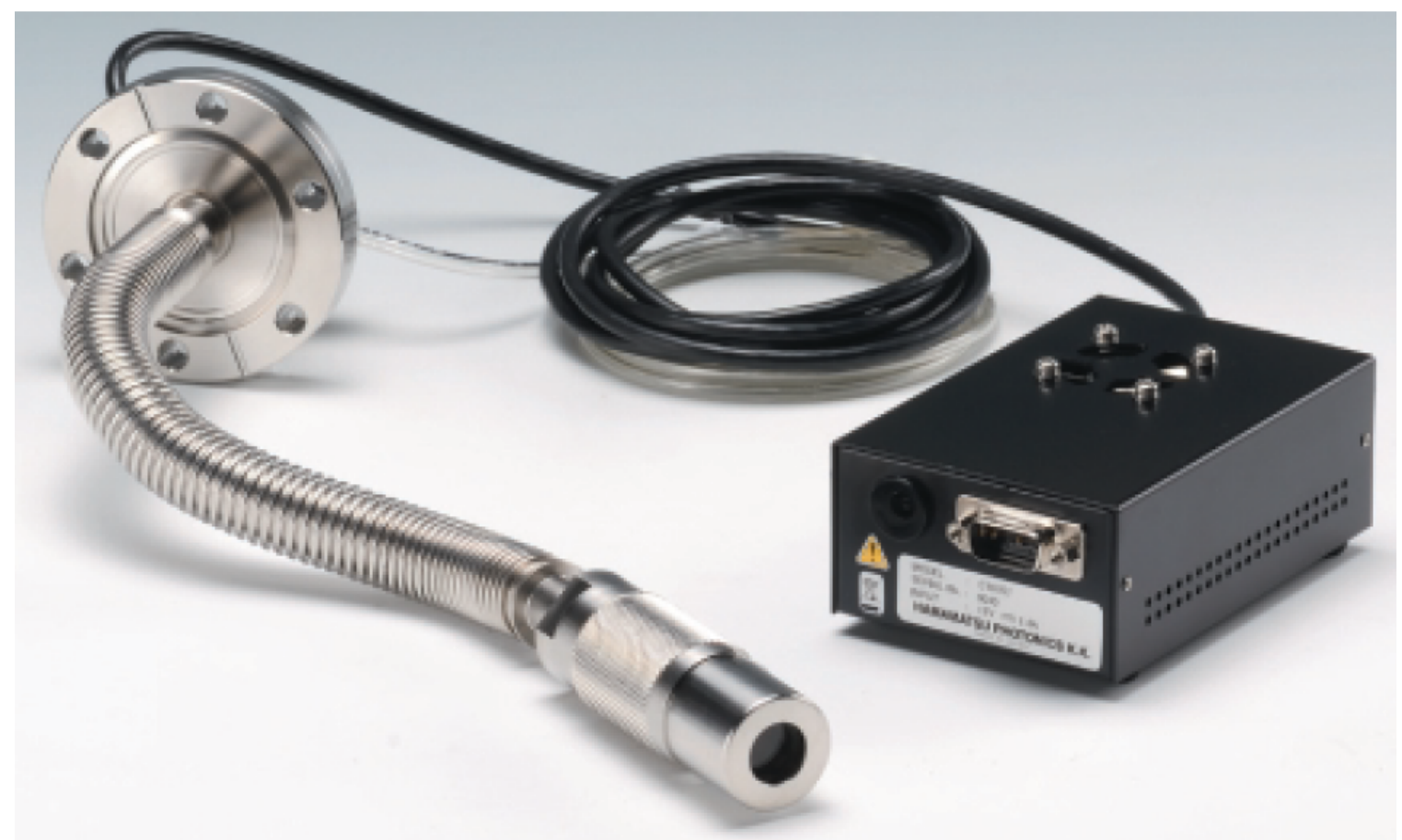

Figure 13. Photograph of the Hamamatsu L10706 Deuterium lamp and power supply. ${ }^{27}$

The manufacturer supplied data of the expected spectral irradiance and directivity.

The spectral irradiance data was collected by the manufacturer using to determine the lamp's irradiance at $50 \mathrm{~cm}$; this data is shown in Fig. 14. Data was also provided on the 
lamp's directivity divergence, showing an average dispersion of $\sim 7.5^{\circ}$. This is a difficult number to quantify as the lamp's output is asymmetrical around the two axial collinear planes which were measured.

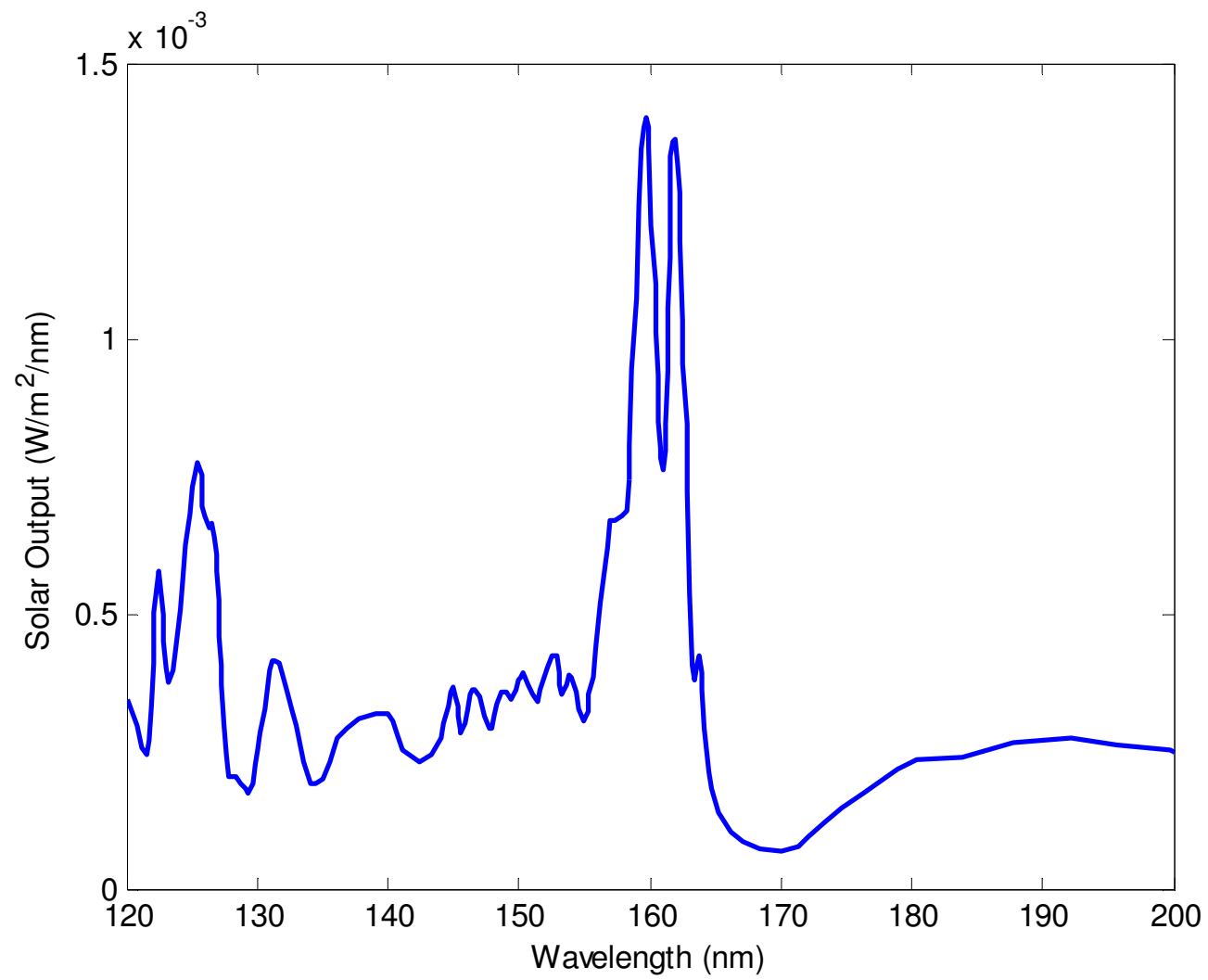

Fig 14. Manufacturer's expected spectral distribution of the L10706 deuterium lamp at 50 $\mathrm{cm}$.

The VUV regime from $100-200 \mathrm{~nm}$ is only a small portion of the total solar output, accounting for a total of $0.104 \mathrm{~W} / \mathrm{m}^{2}$ of the average solar output of $1366.1 \mathrm{~W} / \mathrm{m}^{2}$, or $0.0076 \% .^{15}$ The entire UV regime for everything below $400 \mathrm{~nm}$ contributes 107.43 $\mathrm{W} / \mathrm{m}^{2}$, or $7.86 \%$ of the average solar output. Although VUV is only a small fraction of the UV regimes, it is still one of the most important to study. As discussed in Chapter II, a shorter wavelength means a more energetic the photon that is capable of breaking a stronger bond. So although simulation of the MUV and NUV regimes would apply more 
wattage to a sample, no damage will be observed if the test material was only susceptible to damage from VUV radiation. As a hypothetical example, if all the bonds in a material had a strength of $10 \mathrm{eV}$, no amount of MUV or NUV radiation would cause erosion, as the maximum energy of MUV and NUV radiation is $6.2 \mathrm{eV}$. Equation (1) showed that VUV radiation can break any bond weaker than $12.4 \mathrm{eV}$; however, the lower wattage means that weaker bonds normally affect by longer wavelength UV will be damaged at a slower rate.

Final assembly of the apparatus can be seen in Fig. 15. The cylindrical pyrex bell jar has been removed for clarity; however, the hoist was lowered in order to position the AO and VUV apparatuses in their actual test locations.

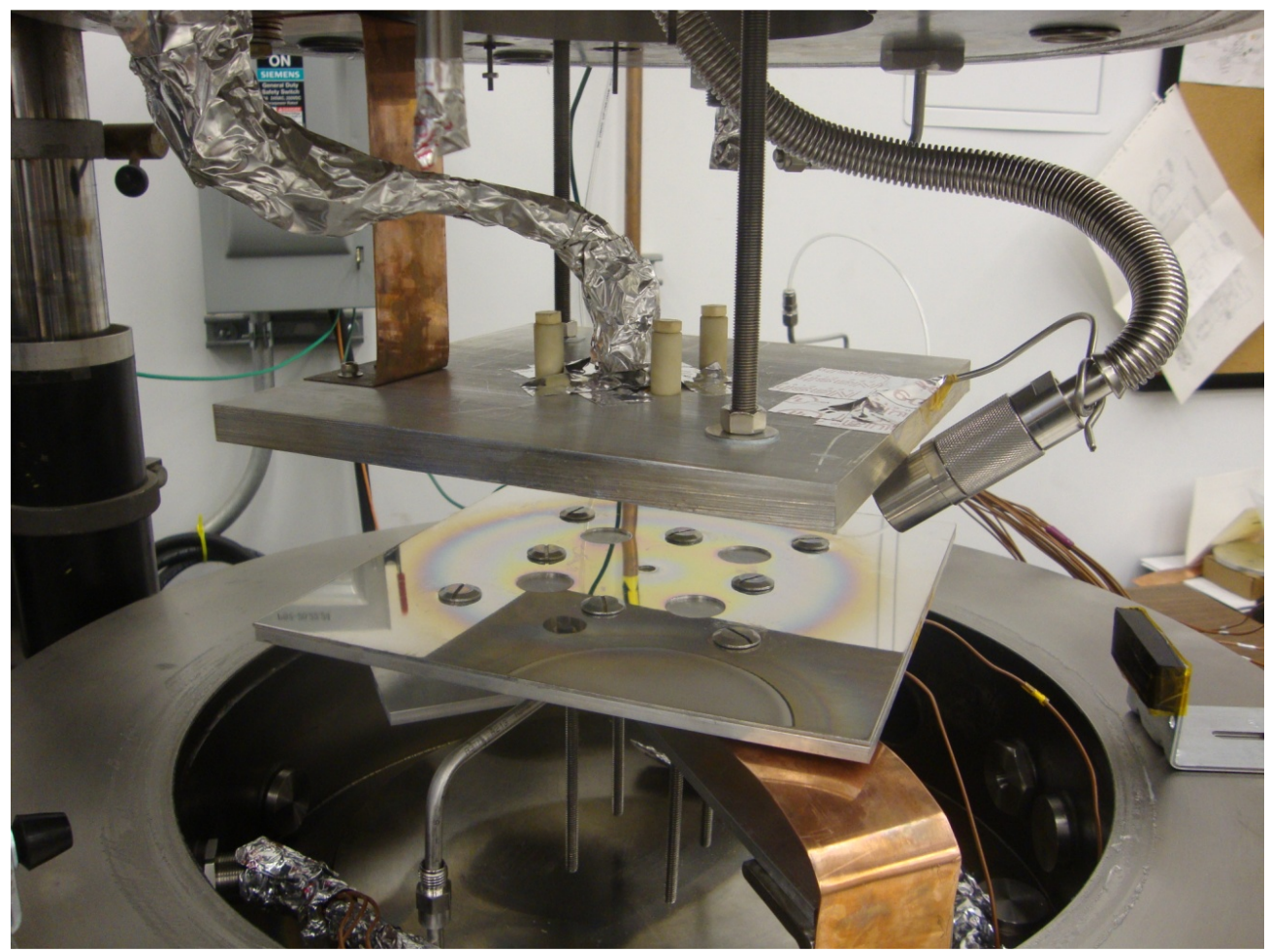

Figure 15. Photograph of the chamber with AO apparatus and VUV light source installed; bell jar not included.

Operational procedures of the AO apparatus and VUV lamp can be found in Appendix B and C. 


\section{System Calibration}

Prior to testing, numerous modifications and upgrades were made to the CCP AO apparatus in order to find a region of stable operation. After numerous attempts at dark space shields, ground paths, mounting mechanisms, and electrical connections the apparatus described in Chapter IV was finalized.

The last step was manual adjusting the AT3 matching network for testing. This process involved disassembling the matchbox in order to adjust the load and tune mechanisms. The load mechanism has one large variable capacitor in line with three fixed capacitors. Depending on the response of the plasma system, capacitance limits were occasionally reached. This required either the addition or removal of the fixed capacitors by bypassing them with $1 \mathrm{~cm}$ silver straps. For the tune mechanism, the seven turn silver inductor had to be varied. This was done by connecting copper straps between turns of the inductor; minor adjustments were also made by either contracting or expanding the length of the inductor. A final configuration, which satisfied all of the expected operational points, was found; this configuration disconnected the three fixed capacitors on the load mechanism while the inductor on the tune mechanism was only slightly compressed in the axial direction.

After this was completed, a small test matrix was designed in order to empirically locate and select the operational characteristics for future testing. It was decided to perform six tests, each for four hours. The test matrix included three gap distances: 5.08, 7.62, and $10.16 \mathrm{~cm}$, and two power levels, 125 and 250 Watts. The gap distances were selected based on the possible configurations which could be set up within the constraints of the chamber. Preliminary testing between 250 and 300 Watts of RF power raised 
concerns about overheating the samples, for this reason the lower power level was chosen to be included in the test matrix.

Air was bled into the system in order to maintain a pressure of $175+/-10$ mTorr with the use of a needle valve. This pressure was chosen since it could maintain a stable plasma across the entire test matrix. No flow meter was available whose range was high enough to determine how many standard cubic centimeters per minute, sccm, were entering the system, but it is estimated to be between 25 and $40 \mathrm{sccm}$.

The final user selected parameter was maximum test temperature. Two type K thermocouples were attached to the bottom of the aluminum ground plate; one was located near the center of the plate with the second near the edge. As high temperatures can alter the reaction efficiency ${ }^{2}$ and may even melt certain polymers, a temperature most similar the expected space environment is desired. The LDEF spacecraft, which collected much of orbital data for spacecraft materials, was shown to have surface temperatures of $\sim 88^{\circ} \mathrm{C} .{ }^{28}$ Ergo, a temperature of $90^{\circ} \mathrm{C}$ was selected, this value was chosen since it did not seem unreasonable for spacecraft operations but still allowed for sufficient AO creation. For the diagnostic tests, tests were not aborted until surpassing $100^{\circ} \mathrm{C}$

After the tests were performed, the samples were analyzed using the mass loss calculation methods described in ASTM standard E2089. Due to availability, only one of the four recommended witness materials was used in the study: 5 mil Kapton HN which was supplied by Sheldahl brand materials. The samples were continuously outgassed for no less than 48 hours at a pressure less than 200 mTorr. As recommended in the standard, a milligram scale was used for weighing, and all measurements were made within five minutes of being removed from the test or outgassing chambers. 
The calculations established in the standard are fairly straightforward. Using the reaction efficiency of Kapton HN stated in the ASTM standard, the equation

$$
f=\frac{\Delta M}{A * \rho * E * t}
$$

is used to find the effective flux, $f$, in atoms $/ \mathrm{cm}^{2}$. The change in mass, $\Delta \mathrm{M}$, is reported in grams, $\mathrm{A}$ is the exposed area, $5.06+/-0.02 \mathrm{~cm}^{2}$, the density, $\rho$, which is $1.435+/-0.002$ $\mathrm{g} / \mathrm{cm}^{3}$, the reaction efficiency of Kapton, E, $3.00+/-0.07 \cdot 10^{-24} \mathrm{~cm}^{3} /$ atom, and the test time, $\mathrm{t}$, in seconds.

By multiplying the total test time, the effective fluence, F, can be solved for using

$$
F=\frac{\Delta M}{A * \rho * E}
$$

The sample film was first cut into $5.08 \mathrm{~cm}$ squares each weighing $\sim 0.475$ grams before being placed between the ground plate and the sample containment cover. The results from the first six diagnostics tests can be seen in Table 3.

\begin{tabular}{|c|c|c|c|c|c|c|c|}
\hline Test & $\begin{array}{l}\text { Gap } \\
(\mathrm{cm})\end{array}$ & $\begin{array}{l}\text { Power } \\
\text { (W) }\end{array}$ & $\begin{array}{l}\text { Temp. } \\
\left({ }^{\circ} \mathrm{C}\right)\end{array}$ & Sample & $\Delta \mathrm{M}(\mathrm{g})$ & $\begin{array}{l}\text { Effective flux } \\
\text { (atoms } / \mathrm{cm}^{2} / \mathrm{s} \text { ) }\end{array}$ & $\begin{array}{c}\text { Effective fluence } \\
\text { (atoms } / \mathrm{cm}^{2} \text { ) }\end{array}$ \\
\hline \multirow{2}{*}{1} & \multirow{2}{*}{5.08} & \multirow{2}{*}{125} & \multirow{2}{*}{88.5} & 1 & 0.010 & $3.185 \cdot 10^{16}$ & $4.586 \cdot 10^{20}$ \\
\hline & & & & 2 & 0.007 & $2.229 \cdot 10^{16}$ & $3.210 \cdot 10^{20}$ \\
\hline 2 & 5.08 & 250 & \multicolumn{5}{|c|}{ Aborted due to excessive temperature } \\
\hline \multirow{2}{*}{3} & \multirow{2}{*}{7.62} & \multirow{2}{*}{125} & \multirow{2}{*}{78.2} & 1 & 0.007 & $2.229 \cdot 10^{16}$ & $3.210 \cdot 10^{20}$ \\
\hline & & & & 2 & 0.006 & $1.911 \mathrm{E} \cdot 10^{16}$ & $2.752 \cdot 10^{20}$ \\
\hline 4 & 7.62 & 250 & \multicolumn{5}{|c|}{ Aborted due to excessive temperature } \\
\hline \multirow{2}{*}{5} & \multirow{2}{*}{10.16} & \multirow{2}{*}{125} & \multirow{2}{*}{74.3} & 1 & 0.005 & $1.592 \cdot 10^{16}$ & $2.293 \cdot 10^{20}$ \\
\hline & & & & 2 & 0.007 & $2.229 \cdot 10^{16}$ & $3.210 \cdot 10^{20}$ \\
\hline 6 & 10.16 & 250 & \multicolumn{5}{|c|}{ Aborted due to excessive temperature } \\
\hline
\end{tabular}

Table 3. Results from diagnostic testing of the AO apparatus. 
These tests were only performed to determine the nominal operation conditions for the apparatus. The results were looked at comparatively across all tests cases and the operational point was determined by relative performance. A discussion with regard to the value and meaning of these results was reserved for Chapter VI.

In order to calibrate a VUV light source, typically a Cesium-Iodide phototube is used; however, this device requires use of a picoammeter and the entire assembly could not be afforded. Based on manufacture's expected irradiance and divergence angles, calculations were done to find the equivalent sun power of the lamp with respect to distance from sample, seen in Fig 16.

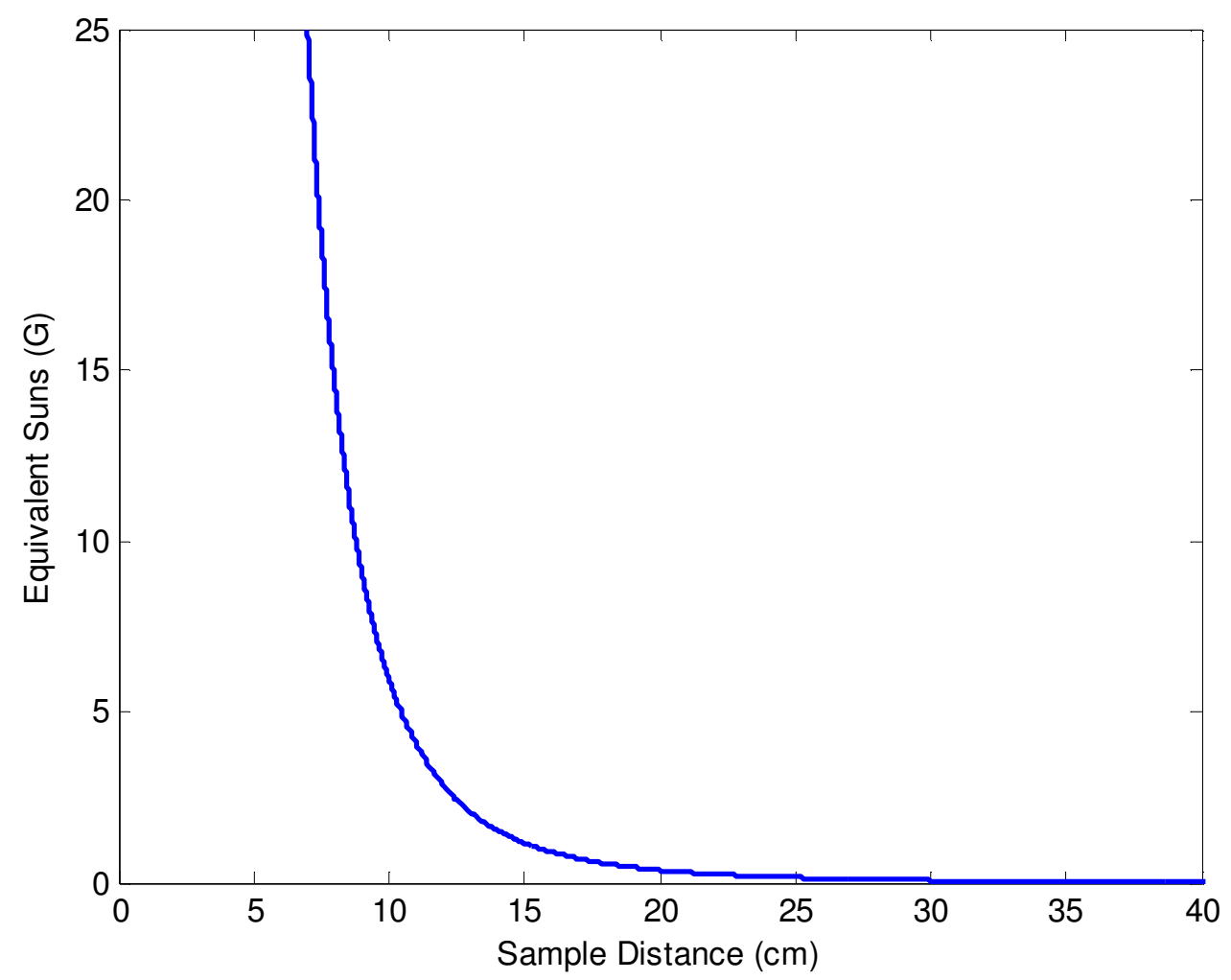

Figure 16. Equivalent Sun power of the L10706 deuterium lamp based on sample distance.

This figure was created using the manufacture's data presented in Chapter III. The irradiance of the light source was transformed through the assumption that the light 
source acts a point source, meaning that the irradiance changes with respect to a $1 / \mathrm{r}^{2}$ law, where $r$ is the distance from the light source to the target. In actuality the lamp resides in tubular structure made of $\mathrm{MgF}_{2}$, and does not act as a point source. Study of similar lamp actually showed that the characteristic relationship was $1 / \mathrm{r}^{2.5} ;{ }^{14}$ however, as no phototube was available to verify this, the point source assumption was employed. In order to calculate the equivalent sun power, the irradiance data for the lamp was integrated across wavelength from 120 to $200 \mathrm{~nm}$ in order to find the total $\mathrm{W} / \mathrm{m}^{2}$ emitted by the lamp. A correction factor was included as the goal is to have a certain equivalent sun power on the $5.06 \mathrm{~cm}^{2}$ sample area, as opposed to the entire illumination area of the lamp. This factor is a ratio of the sample area divided by the illumination area. The same integration process was conducted for the solar irradiance data from ASTM E0490; equivalent sun power is simply the ratio of these two values.

As mentioned before, the desired equivalent sun power was between 3 and $5 \mathrm{G}$. The trend from Fig. 16 was used to determine the distance at which the lamp was placed from the samples. Margin was applied for an uncertainty in positioning the lamp in the chamber, and a goal value of $4.5 \mathrm{G}$ was selected. From the calculations, the lamp placement was determined to be $10.72 \mathrm{~cm}$ from the sample location.

A comparison was made between the AM0 and L10706 spectral irradiance data. As mentioned in Chapter III, it is important to understand where the peaks of the spectral output are situated. The spectral irradiance of the L10706 was plotted as if it operating at 1 equivalent sun, a sample distance of $15.62 \mathrm{~cm}$. This plot can be seen in Fig. 17, which also includes the irradiance of the L10706 at the desired test distance of $10.72 \mathrm{~cm}$. At this distance the total illumination of the lamp is $6.26 \mathrm{~cm}^{2}$, or a circle with a diameter of 2.82 
$\mathrm{cm}$. This allows for some margin if the pointing accuracy of the lamps as the target sample area is a $2.54 \mathrm{~cm}$ diameter circle.

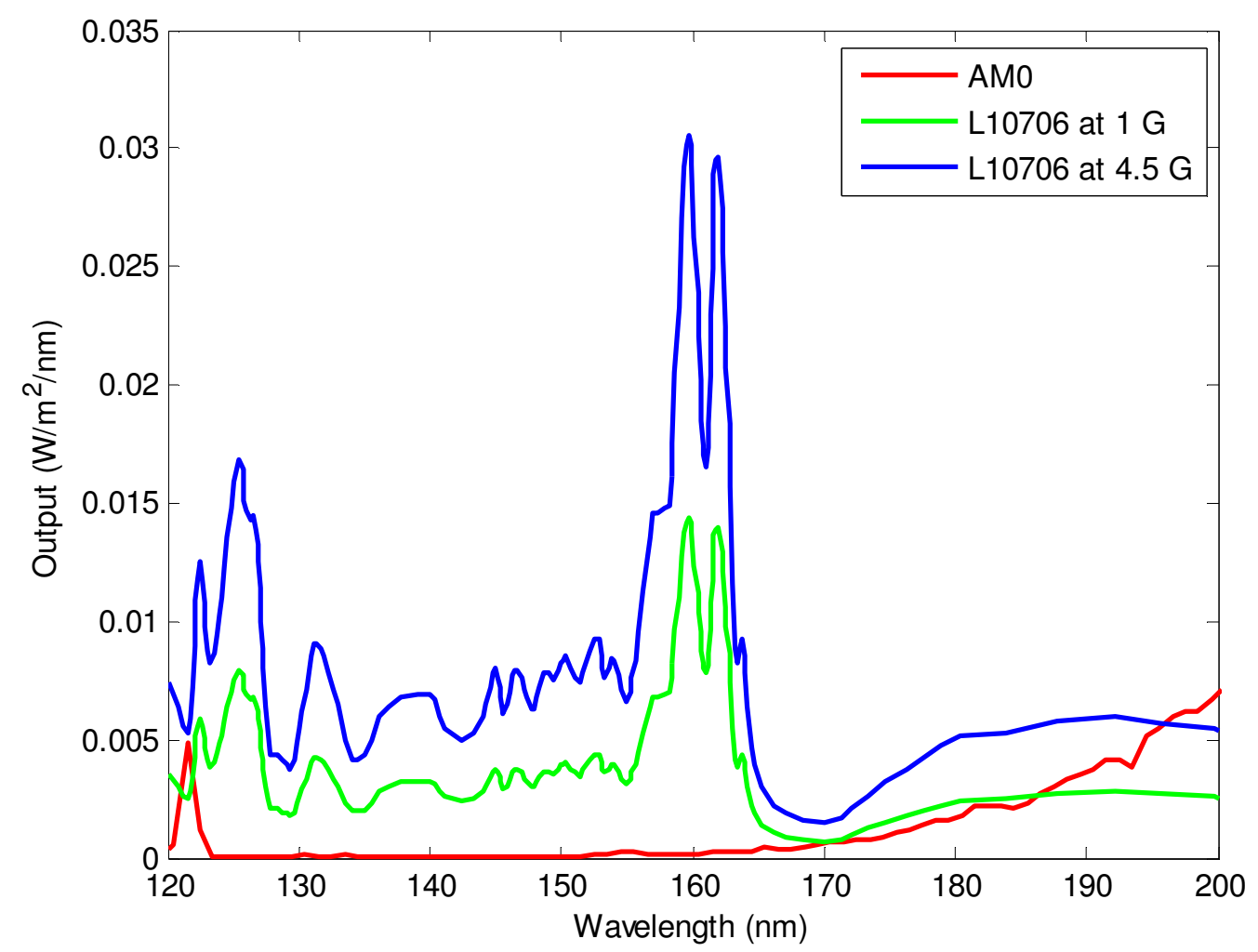

Figure 17. Spectral irradiance for AM0 solar output and the L10706 operating at 1 and 4.5 equivalent suns.

Note that the L10706 deuterium lamp has a peak output at 160nm which is greater than the solar output. This means that any material that readily absorbs $160 \mathrm{~nm}$ radiation will erode faster. On the contrary, the AM0 data has a peak at $121 \mathrm{~nm}$, the Lyman-alpha line of hydrogen, meaning that a material which absorbs at this wavelength will erode slower in the test chamber. In general, the L10706 has a higher output than the sun from $120-165 \mathrm{~nm}$, while the solar output is greater above $165 \mathrm{~nm}$. It is important to understand these relationships and not to be misled by the fact that these spectral distributions have an equal total output of $0.104 \mathrm{~W} / \mathrm{m}^{2}$ on the $5.06 \mathrm{~cm}^{2}$ sample cell area. 


\section{Results and Validation}

Based on the results of the diagnostic test it was decided to set the nominal operational configuration at a gap distance of $7.62 \mathrm{~cm}$ with a power level of $125 \mathrm{Watts}$. A photograph of the plasma with similar operational conditions can be seen in Fig. 18. This point was chosen since it provides the highest AO flux without approaching the maximum temperature limit. The final configuration of the UV lamp was at the desired distance of $10.7 \mathrm{~cm}$ at an angle of $\sim 45^{\circ}$ from the sample; the lamp could not be positioned normal to the sample due to interference with the AO apparatus. As this is the configuration to be used for future research and evaluation long durations tests were performed to ensure that the system could operate continuously.

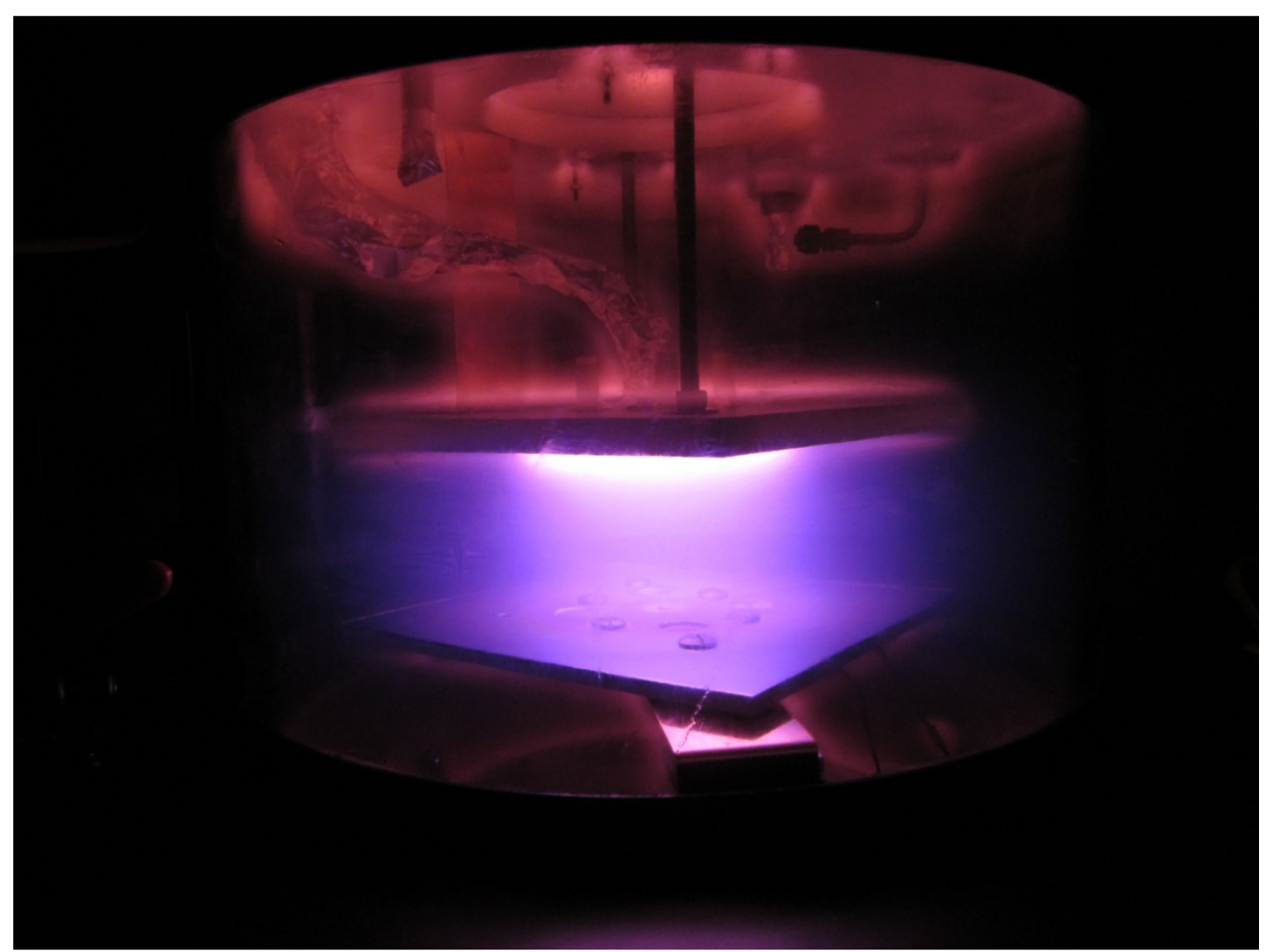

Figure 18. Photograph of the AO plasma operating at the long duration test configuration. 
This photograph shows the strong primary emission in-between the two electrodes, which is comprised of highly excited nitrogen and AO. Above and below the electrodes lie secondary emissions that consist of lower energy nitrogen ions. It is difficult to eliminate these secondary emissions, and since they do not affect the test center or operation of the system little effort beyond the DSS was made to eliminate this superfluous plasma.

The tests were conducted over 24 hours, in which the average mass loss of the Kapton $\mathrm{HN}$ was $0.032+/-0.001 \mathrm{~g}$. In order to determine the effective flux and fluence the same analysis described in Chapter V was performed with one addition. The ASTM E2089 standard, which is used to compare AO erosion data across multiple facilities, states a reaction efficiency of Kapton $\mathrm{HN}$ as $3.00 \cdot 10^{-24} \mathrm{~cm}^{3} /$ atom. This standard was reapproved in 2006; however, since then analysis has been performed on samples that were returned from the ISS as part of the MISSE Polymer Erosion and Contamination Experiment. This experiment returned a slightly different value of $2.81+/-0.07 \cdot 10^{-24}$ $\mathrm{cm}^{3} /$ atom that was used in a secondary analysis. ${ }^{3}$ Results can be seen in Table 4.

The VUV lamp is only able to illuminate one sample at a time, and was focused on sample \#4 for the duration of test \#2 and \#3. As these samples were exposed to the VUV radiation and experienced accelerated erosion due to the synergistic affects of $\mathrm{AO}$ and VUV, the values shown for the effective flux and fluence were not based on the mass loss of these samples. The values are an approximation based upon the three nearby witness samples of their respective test groups; they are simply an average of the calculated numbers. As explained in Chapter $\mathrm{V}$, the equivalent sun power was approximately 4.5 resulting in 108 ESHs during the 24 hour test. 
Table 4. Results from long duration AO and VUV exposure.

\begin{tabular}{|c|c|c|c|c|c|c|}
\hline Test & Sample & $\Delta \mathrm{M}(\mathrm{g})$ & $\begin{array}{c}\text { ASTM E2089 } \\
\text { Effective flux } \\
\text { (atoms } / \mathrm{cm}^{2} / \mathrm{s} \text { ) }\end{array}$ & $\begin{array}{l}\text { ASTM E2089 } \\
\text { Effective } \\
\text { fluence } \\
\text { (atoms } / \mathrm{cm}^{2} \text { ) }\end{array}$ & $\begin{array}{l}\text { MISSE Effective } \\
\text { flux } \\
\text { (atoms } / \mathrm{cm}^{2} / \mathrm{s} \text { ) }\end{array}$ & $\begin{array}{l}\text { MISSE Effective } \\
\text { fluence } \\
\text { (atoms } / \mathrm{cm}^{2} \text { ) }\end{array}$ \\
\hline \multirow{4}{*}{1} & 1 & $\begin{array}{c}0.032 \\
+/-0.002\end{array}$ & $\begin{array}{c}1.698 \\
+/-0.156 \cdot 10^{16}\end{array}$ & $\begin{array}{c}1.467 \\
+/-0.134 \cdot 10^{21}\end{array}$ & $\begin{array}{c}1.813 \\
+/-0.169 \cdot 10^{16}\end{array}$ & $\begin{array}{c}1.567 \\
+/-0.145 \cdot 10^{21}\end{array}$ \\
\hline & 2 & $\begin{array}{c}0.033 \\
+/-0.002\end{array}$ & $\begin{array}{c}1.751 \\
+/-0.158 \cdot 10^{16}\end{array}$ & $\begin{array}{c}1.513 \\
+/-0.135 \cdot 10^{21}\end{array}$ & $\begin{array}{c}1.870 \\
+/-0.171 \cdot 10^{16}\end{array}$ & $\begin{array}{c}1.616 \\
+/-0.147 \cdot 10^{21}\end{array}$ \\
\hline & 3 & $\begin{array}{c}0.032 \\
+/-0.002 \\
\end{array}$ & $\begin{array}{c}1.698 \\
+/-0.156 \cdot 10^{16}\end{array}$ & $\begin{array}{c}1.467 \\
+/-0.134 \cdot 10^{21}\end{array}$ & $\begin{array}{c}1.813 \\
+/-0.169 \cdot 10^{16}\end{array}$ & $\begin{array}{c}1.567 \\
+/-0.145 \cdot 10^{21}\end{array}$ \\
\hline & 4 & $\begin{array}{c}0.034 \\
+/-0.002\end{array}$ & $\begin{array}{c}1.804 \\
+/-0.159 \cdot 10^{16}\end{array}$ & $\begin{array}{c}1.559 \\
+/-0.136 \cdot 10^{21}\end{array}$ & $\begin{array}{c}1.664 \\
+/-0.173 \cdot 10^{16}\end{array}$ & $\begin{array}{c}1.665 \\
+/-0.148 \cdot 10^{21}\end{array}$ \\
\hline \multirow{4}{*}{2} & 1 & $\begin{array}{c}0.033 \\
+/-0.002\end{array}$ & $\begin{array}{c}1.751 \\
+/-0.158 \cdot 10^{16}\end{array}$ & $\begin{array}{c}1.513 \\
+/-0.135 \cdot 10^{21}\end{array}$ & $\begin{array}{c}1.870 \\
+/-0.171 \cdot 10^{16}\end{array}$ & $\begin{array}{c}1.616 \\
+/-0.147 \cdot 10^{21}\end{array}$ \\
\hline & 2 & $\begin{array}{c}0.032 \\
+/-0.002 \\
\end{array}$ & $\begin{array}{c}1.698 \\
+/-0.156 \cdot 10^{16}\end{array}$ & $\begin{array}{c}1.467 \\
+/-0.134 \cdot 10^{21}\end{array}$ & $\begin{array}{c}1.813 \\
+/-0.169 \cdot 10^{16} \\
\end{array}$ & $\begin{array}{c}1.567 \\
+/-0.145 \cdot 10^{21} \\
\end{array}$ \\
\hline & 3 & $\begin{array}{c}0.033 \\
+/-0.002 \\
\end{array}$ & $\begin{array}{c}1.751 \\
+/-0.158 \cdot 10^{16}\end{array}$ & $\begin{array}{c}1.513 \\
+/-0.135 \cdot 10^{21}\end{array}$ & $\begin{array}{c}1.870 \\
+/-0.171 \cdot 10^{16}\end{array}$ & $\begin{array}{c}1.616 \\
+/-0.147 \cdot 10^{21}\end{array}$ \\
\hline & $4^{*}$ & $\begin{array}{c}0.036 \\
+/-0.002 \\
\end{array}$ & $\begin{array}{c}1.734 \\
+/-0.031 \cdot 10^{16} \\
\end{array}$ & $\begin{array}{c}1.498 \\
+/-0.026 \cdot 10^{21} \\
\end{array}$ & $\begin{array}{c}1.851 \\
+/-0.033 \cdot 10^{16} \\
\end{array}$ & $\begin{array}{c}1.599 \\
+/-0.028 \cdot 10^{21} \\
\end{array}$ \\
\hline \multirow{4}{*}{3} & 1 & $\begin{array}{c}0.031 \\
+/-0.002\end{array}$ & $\begin{array}{c}1.645 \\
+/-0.154 \cdot 10^{16}\end{array}$ & $\begin{array}{c}1.422 \\
+/-0.132 \cdot 10^{21}\end{array}$ & $\begin{array}{c}1.757 \\
+/-0.168 \cdot 10^{16}\end{array}$ & $\begin{array}{c}1.518 \\
+/-0.144 \cdot 10^{21}\end{array}$ \\
\hline & 2 & $\begin{array}{c}0.030 \\
+/-0.002\end{array}$ & $\begin{array}{c}1.592 \\
+/-0.153 \cdot 10^{16}\end{array}$ & $\begin{array}{c}1.376 \\
+/-0.131 \cdot 10^{21}\end{array}$ & $\begin{array}{c}1.700 \\
+/-0.166 \cdot 10^{16}\end{array}$ & $\begin{array}{c}1.469 \\
+/-0.142 \cdot 10^{21}\end{array}$ \\
\hline & 3 & $\begin{array}{c}0.030 \\
+/-0.002 \\
\end{array}$ & $\begin{array}{c}1.592 \\
+/-0.153 \cdot 10^{16} \\
\end{array}$ & $\begin{array}{c}1.376 \\
+/-0.131 \cdot 10^{21} \\
\end{array}$ & $\begin{array}{c}1.700 \\
+/-0.166 \cdot 10^{16} \\
\end{array}$ & $\begin{array}{c}1.469 \\
+/-0.142 \cdot 10^{21}\end{array}$ \\
\hline & $4^{*}$ & $\begin{array}{c}0.035 \\
+/-0.002\end{array}$ & $\begin{array}{c}1.610 \\
+/-0.031 \cdot 10^{16}\end{array}$ & $\begin{array}{c}1.391 \\
+/-0.026 \cdot 10^{21}\end{array}$ & $\begin{array}{c}1.719 \\
+/-0.033 \cdot 10^{16}\end{array}$ & $\begin{array}{c}1.485 \\
+/-0.028 \cdot 10^{21}\end{array}$ \\
\hline
\end{tabular}

*Exposed to VUV light source, flux an fluence values are test averages

The steady state temperatures during these tests remained within the defined constraints, the average steady state temperatures for tests \#1-3 were $83.7^{\circ} \mathrm{C}, 84.4^{\circ} \mathrm{C}$, and $87.4^{\circ} \mathrm{C}$ respectively. The results from 24 hour tests show an average fluence of $1.47+/-$ $0.06 \cdot 10^{21}$ atoms $/ \mathrm{cm}^{2}$ based on ASTM E2089. As discussed in Chapter II, it is hard to quantify the equivalent on orbit exposure time due to the affects of the solar cycle and orbital location, but this fluence would equate to an orbital exposure on the order of 
weeks to months. This result demonstrates the ability of this apparatus to provide accelerated simulation of the LEO environment.

Results from the sample exposed to VUV radiation and AO show an increased mass loss when compared to the samples only exposed to $\mathrm{AO}, 10.1 \%$ percent greater for test \#2 and $15.4 \%$ greater for test \#3. As a control, a single sample of Kapton HN was exposed to the VUV source in the exact configuration used during the combined testing. This sample showed a negligible mass loss that was within the error margin of the scale. These results demonstrate that VUV and AO do indeed act synergistically, and that simultaneous exposure increases the erosion rates of materials.

Although these results match expectations established by background research and methodologies described in ASTM E2089, it is important to provide further verification as this is a unique apparatus that is being calibrated for future material studies. The simplest method of verification is based upon the reaction of Kapton HN to the simulated environment. As expected, the Kapton film lost mass, as seen in Table 4, and became less reflective. This was qualitatively observed, as the sample exposure areas visibility became less reflected and developed and opaque surface finish. Photographs of an exposed sample can be seen in Fig. 19. 


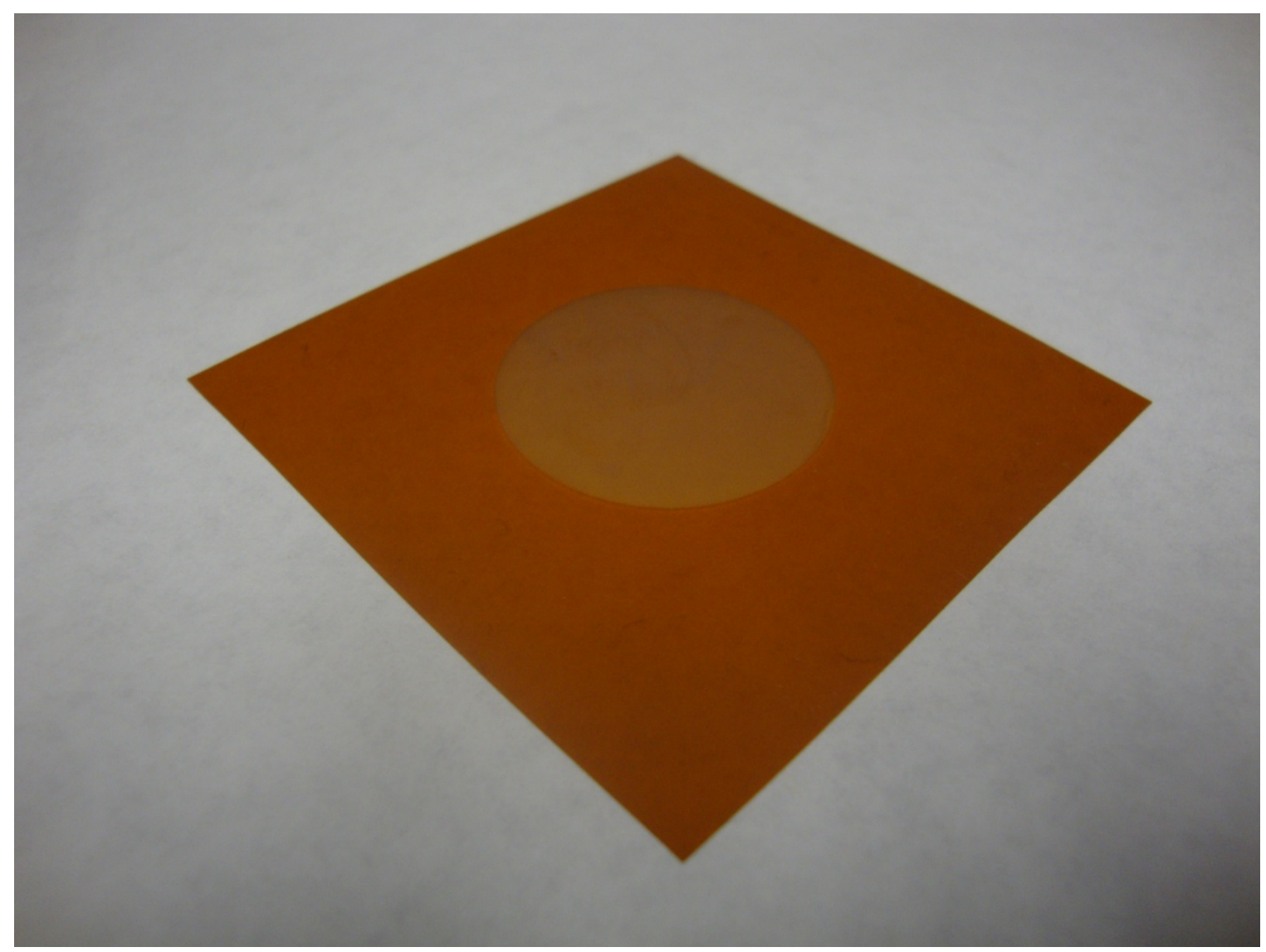

Figure 19. Photograph of a Kapton HN sample subjected to a 24 hour exposure.

Examination of the exposure area also showed a distinct boundary between the exposed and non-exposed portions, verification that the sample containment cover plate was providing adequate control of the exposure area.

In order to quantitatively measure the decreased reflectance of the samples spectral measurements were performed. A spectrometer manufactured by Analytic Spectral Devices Inc. that is on loan from NASA Johnson Space Center was used to measure the absolute reflectance of the samples. The FieldSpec Spectrometer, model FSP 350-2500P, has 717 channels which can measure a range from 350 to $2500 \mathrm{~nm}$ at a resolving power of approximately 200. The resolving power equates to a bandwidth of 10 $\mathrm{nm}$ at two microns, while an ample number of channels is advantageous as it diminishes the degradation of the spectral resolution. 
A comparison of the spectral response of nominal Kapton $\mathrm{HN}$ and a sample which was exposed to the AO for 24 hours can be seen in Fig. 20.

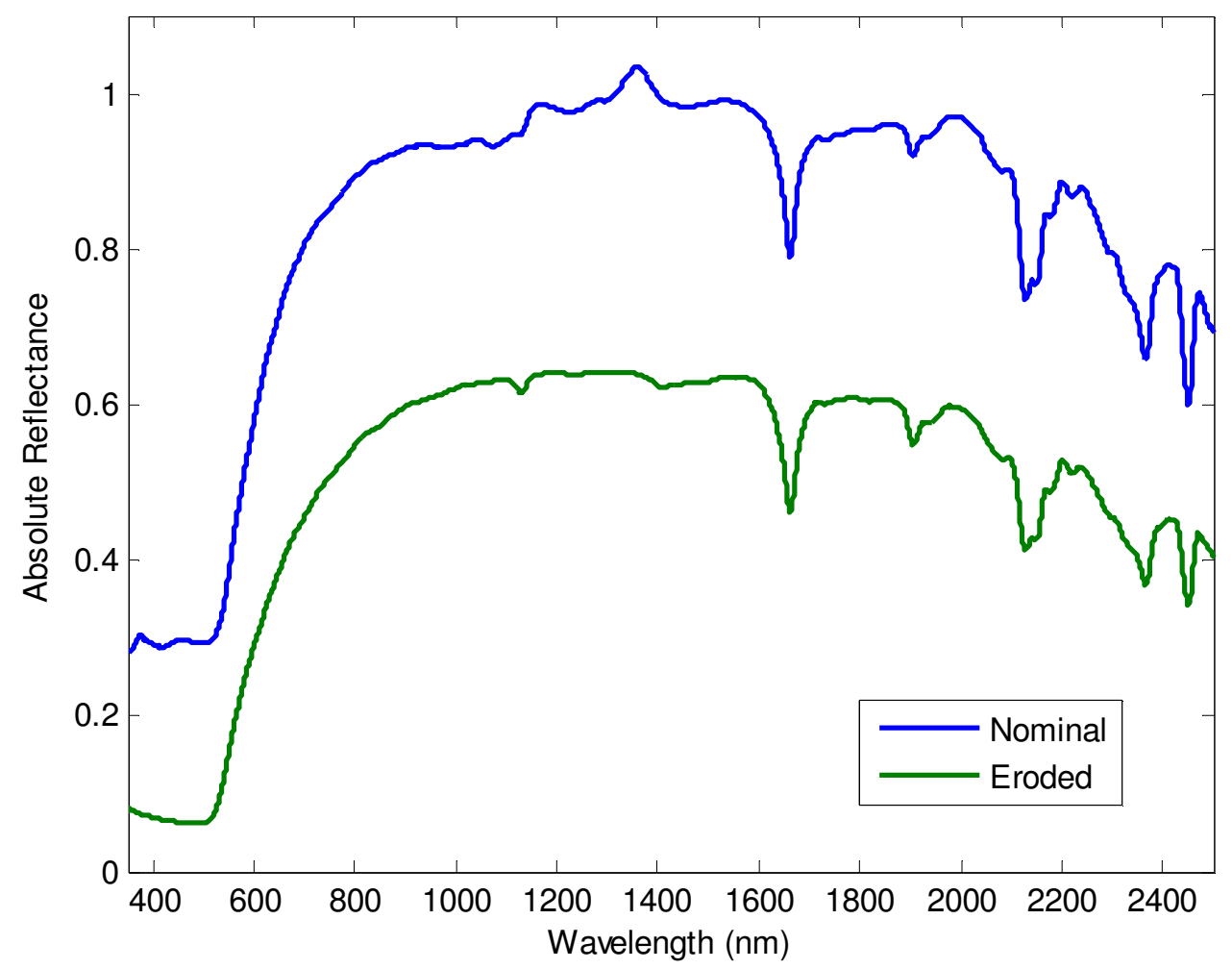

Figure 20. Spectral comparison between nominal and eroded Kapton HN.

It is evident that the eroded sample is less reflective, with an average decrease of $33 \%$ in the reflective response. The nominal Kapton is fairly reflective, and even shows a reflective response greater than one near $1360 \mathrm{~nm}$. The ASD spectrometer functions by measuring the diffuse reflectance of the sample. In this case, the specular component of Kapton HN's reflection is greater than the reference spectralon used in calibration of the spectrometer. This behavior is not a concern, as the emphasis is on the relative change in material properties.

The final method used to verify the presence of AO manifested conclusive results. Using the same spectrometer, the plasma between the two electrodes was evaluated at the 
test case with the goal of identifying the spectral lines of AO. Using the National Institute of Standards and Technology's, NIST, Atomic Spectra Database it was found that the most intense emission lines for $\mathrm{AO}$ were located at near 777.3 and $844.6 \mathrm{~nm} ;^{20}$ identifying these lines would prove the presence of AO.

The first measurement attempts were made using air as the working gas, meaning $\sim 78 \% \mathrm{~N}_{2}$ and $\sim 21 \% \mathrm{O}_{2}$, however, the spectrometer could only measure relative intensity in the operational mode needed to take data. This meant that although small peaks at 777.3 and $844.6 \mathrm{~nm}$ were present, they were overshadowed by extremely intense emission lines from atomic nitrogen. For this reason the apparatus's operational gas was changed from air to industrial oxygen, $\sim 92 \% \mathrm{O}_{2}$ and $\sim 8 \% \mathrm{~N}_{2}$, in order to lessen the effects of the nitrogen plasma. The spectra output of this plasma can be seen in Fig. 21.

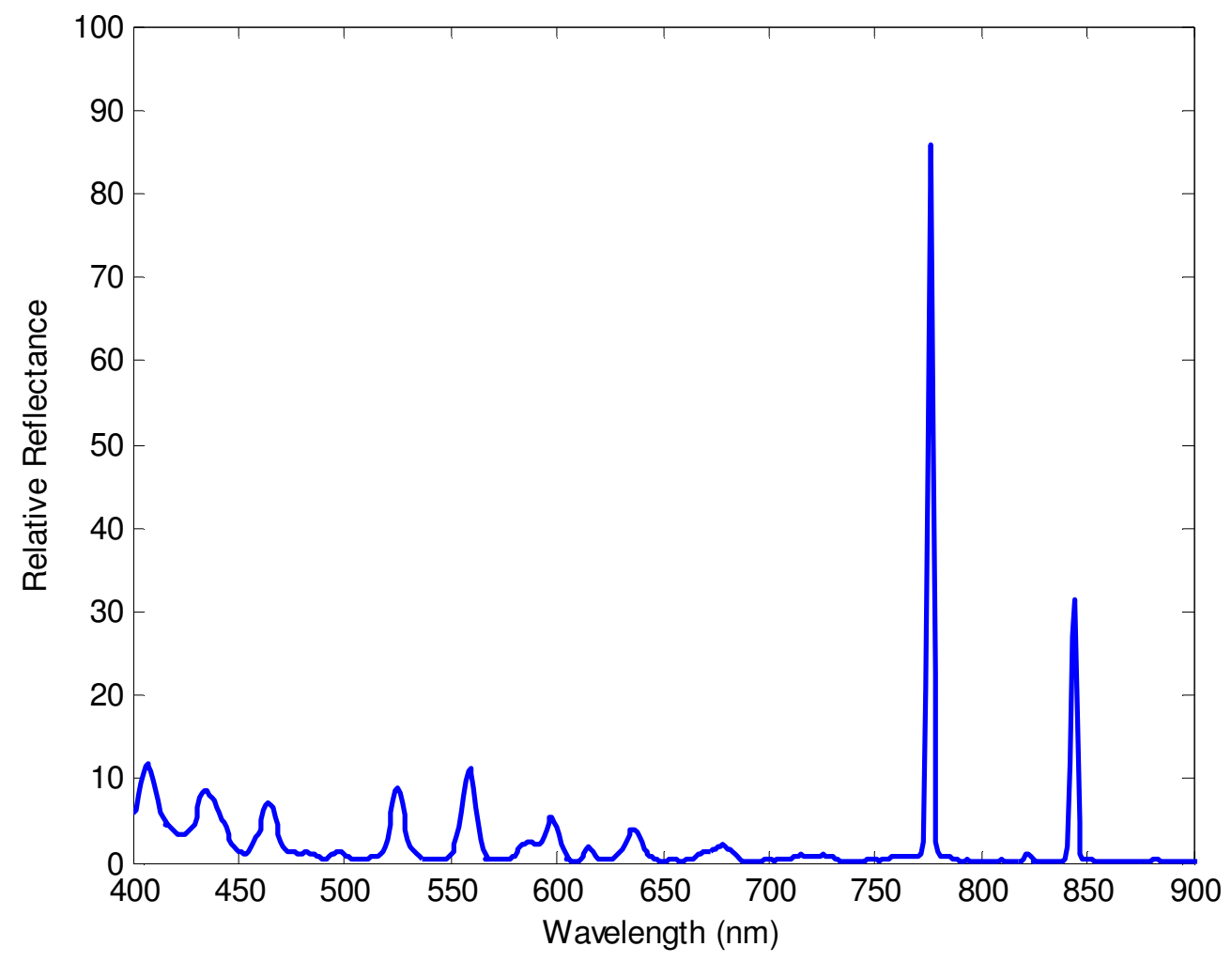

Figure 21. Spectral output of the AO plasma while operating on $\sim 92 \%$ oxygen. 
This plot clearly shows the expected emission lines at 777.3 and $844.6 \mathrm{~nm}$, further proving that $\mathrm{AO}$ actually is created by the apparatus. The numerous peaks at the lower wavelengths can all be attributed to either secondary emission lines of AO or primary emission lines of nitrogen. 


\section{Future Work}

The next objective for this apparatus is to begin experimentation on spacecraft materials to understand their reactions to the LEO environment. A set of spacecraft materials has been donated by Sheldahl Brand materials, including a few materials that would potentially be used in LEO as their coatings are designed to arrest AO erosion. A partial list of these materials includes Kapton films with germanium, aluminum, silver, and silicon-oxides coatings, as well as Beta-cloth and Teflon sheets. The intended test configuration would use all four test cells: two cells for Kapton HN witness samples and two cells for the material in questions. The deuterium lamp could be focused on one of the test samples to provide information on the material's reaction to simultaneous exposure.

There are numerous potential upgrades that could be made to the apparatus. First of which being the addition of a Cesium-Iodide phototube to calibrate the deuterium lamp, eliminating the dependence on the manufacturer's data while getting rid of assumptions made during the calculation of the equivalent solar intensity. Further additions include a second light source to complete the simulation of the UV environment. This would involve the installation of a xenon based lamp to provide radiation in the 200-400 $\mathrm{nm}$ regime. Finally, a cooling mechanism to control the temperature of the substrates would allow added customizability to experimental design. 


\section{Conclusion}

An apparatus has been developed in Cal Poly's space simulation laboratory designed to simulate the LEO environment. The system is capable of exposing materials to Atomic Oxygen and Vacuum Ultraviolet radiation both independently and simultaneously while maintaining a pressure near 175 mTorr. Through short duration diagnostic testing and long duration material erosion experiments, the device has been shown to operate effectively, and is capable of providing accurate and repeatable simulation of the LEO environment.

After the design of the apparatus was finalized, two primary methods were used to verify proper operation. The first method was based upon the reaction of a well characterized material, Kapton HN. The erosion of this material in the apparatus showed ample mass loss as well as an apparent decrease in the material's reflective response; quantitative analysis using a mass spectrometer showed $33 \%$ decrease in the material's absolute reflectivity. The second method involved direct spectral analysis of the generated $\mathrm{AO}$ environment. Using $92 \%$ oxygen as the working gas, the apparatus generated a plasma with intense emission lines at 777.3 and $844.6 \mathrm{~nm}$, congruent with the emission lines for atomic oxygen reported by the National Institute of Standards and Technology.

This was all accomplished by the combination of two devices. The AO generation system operates by disassociating molecular oxygen in a capacitively coupled plasma powered by an $13.56 \mathrm{MHz}, 300 \mathrm{Watt} \mathrm{RF}$ generator. In the nominal configuration the two electrodes are spaced $7.62 \mathrm{~cm}$ apart, while air is introduced between the two plates to sustain a pressure around 175 mTorr. A sample containment cover slide allows for four 
circular samples, $2.54 \mathrm{~cm}$ in diameter, to be placed on the ground electrode. The plasma is maintained at a steady state temperature near $85^{\circ} \mathrm{C}$ while operating on $125 \mathrm{~W}$ of $\mathrm{RF}$ power. The second device is a specialized deuterium lamp outfitted with a MagnesiumFluoride window and is capable of emitting at wavelengths as short as $115 \mathrm{~nm}$. Spectral data provided by the manufacturer helped determine the proper placement of the lamp; in order to obtain 4.5 equivalent sun power over the $120-200 \mathrm{~nm}$ bandwidth, the lamp was placed $10.7 \mathrm{~cm}$ away from the desired test specimen.

The apparatus was shown to provide an AO flux of $1.70+/-0.07 \cdot 10^{16}$ atoms $/ \mathrm{cm}^{2}$; 24 hour tests were performed to simulate long exposure to the LEO environment, and average total fluence of $1.47+/-0.06 \cdot 10^{21}$ atoms $/ \mathrm{cm}^{2}$ was observed. It is difficult to equate this fluence to a finite orbital duration due the variation of the $\mathrm{AO}$ environment with respect to space weather and orbital location; however, this fluence would compare to weeks or months in a typical LEO.

Independent AO experiments showed an average mass loss of $0.032+/-0.001 \mathrm{~g}$, when coupled with VUV radiation, the mass loss increased to $0.0355+/-0.002 \mathrm{~g}$. This confirms expectations that the synergistic affects of AO and VUV increase the erosion rate of materials.

Validation of this apparatus is based upon the need to understand the interactions between spacecraft materials and the space environments. The space environment produces distinctive conditions that have numerous synergistic interactions. Computational models exist but cannot produce concrete results while in-situ material evaluation is costly and cannot provide accelerated exposure. A ground based apparatus 
which can perform these tasks provides a repeatable and accelerated simulation of the space environment.

The apparatus developed in this master's thesis has proven these capabilities, and with the above diagnostics, verification, and validation, this apparatus can now be used for LEO space simulations. This establishes a facility at Cal Poly which is capable of performing environmental studies that can provide valuable information to spacecraft and materials engineers, initiating future experimentation to advance a materials knowledge base and progress the exploration of the space environment. 


\section{References}

${ }^{1}$ Hastings, G., and Garrett, H., Spacecraft Environment Interactions, Cambridge Atmospheric and Space Science Series, Cambridge University Press, Cambridge, United Kingdom, 2004.

${ }^{2}$ Tribble, A.C., The Space Environment: Implications for Spacecraft Design, Princeton University Press, Princeton, New Jersey, 2003.

${ }^{3}$ Mccarthy, C.E., Banks, B.A., and deGroh, K.K., "MISSE 2 PEACE Polymers Experiment Atomic Oxygen Erosion Yield Error Analysis," NASA TM-216903, 2010.

${ }^{4}$ Pisacane, V., The Space Environment at its Effects on Space Systems, AIAA Education Series, AIAA, New York, 2008.

${ }^{5}$ Banks, B.A., Miller, S.K., and de Groh, K.K., "Low Earth Orbital Atomic Oxygen Interactions with Materials," $2^{\text {nd }}$ International Energy Conversion Engineering Conference, AIAA 2004-5638.

${ }^{6}$ Mathworks, Matlab, Software Package, Ver. 2008a, Natick, MA.

${ }^{7}$ Picone, J.M., Hedin, A.E., Prob, D.P., and Aikin, A.C., "NRLMSISE-00 Empirical Model of the Atmosphere: Statistical Comparisons and Scientific Issues," Journal of Geophysical Research, Dec. 2001. DOI: 10.1029/2002JA009430.

${ }^{8}$ Banks, B.A., Rutledge, S.K., Paulsen, P.E., and Steuber, T.J., "Simulation of the Low Earth Orbital Atomic Oxygen Interaction with Materials by Means of an Oxygen Ion Beam,” NASA TM-101971, 1989.

${ }^{9}$ Rutledge, S.K., Banks, B.A., Chichernea, V.A., and Haytas, C.A., "Cleaning of Fire Damaged Watercolor and Textiles Using Atomic Oxygen," NASA TM-210335, 2000.

${ }^{10}$ Banks, B.A., Backus, J.A., Mann, M.V., Waters, D.L., Cameron, K.C., and deGroh, K.K., "Prediction of Atomic Oxygen Erosion Yield for Spacecraft Polymers," Journal of Spacecraft and Rockets, Vol. 48, No. 1, January-February 2011. DOI: 10.2514/1.48849.

${ }^{11}$ Banks, B.A., Waters, D.L., Thorson, S.D., Miller, S.K., deGroh, K.K., and Snyder, A., "Comparison of Atomic Oxygen Erosion Yields at Various Energy and Impact Angles," NASA TM-214363, 2006.

${ }^{12}$ Dever, J.A., Bruckner, E.J., and Rodriguez, E., "Synergistic Effects of Ultraviolet Radiation, Thermal Cycling and Atomic Oxygen on Altered and Coated Kapton Surfaces," AIAA-1992-0794. 
${ }^{13}$ Dworak, D.P., Banks, B.A., Karniotis, C.A., and Soucek, M.D., "Evaluation of Protective Silicone/Siloxane Coatings in Simulated Leo Earth Orbit Environment," Journal of Spacecraft and Rockets, Vol. 43, No. 2, March-April 2006.

${ }^{14}$ Dever, J.A., Pietromica, A.J, Stueber, T.J., Sechkar, E.A., and Messer, R.K., "Simulated Space Vacuum Ultraviolet (VUV) Exposure Testing for Polymer Films," AIAA-2001-1054.

${ }^{15}$ ASTM E 490-00a, "Solar Constant and Zero Air Mass Solar Spectral Irradiance Tables," ASTM International, West Conshohocken, PA. Reapproved in 2006.

${ }^{16}$ Dever, J.A., Banks, B.A., and Yan, L., "Effects of Vacuum Ultraviolet Radiation on DC93-500 Silicone," Journal of Spacecraft and Rockets, Vol. 43. No. 2, March-April 2006.

${ }^{17}$ Banks, B.A., Stueber, T.J., Snyder, S.A., Rutledge, S.K., and Norris, M.J., "Atomic Oxygen Erosion Phenomena," AIAA-97-3903.

${ }^{18}$ Banks, B.A., Rutledge, S.K., de Groh, K.K., Stidman, C.R., Gebauer, L., and LaMoreaux, C.M., "Atomic Oxygen Durability Evaluation of Protected Polymers Using Thermal Energy Plasma Systems” NASA TM-106855, 1993.

${ }^{19}$ Stidham, C.R., Stueber, T.J., Rutledge, S.K., Banks, B.A., Dever, J.A., and Bruckner, E.J., "Low Earth Orbital Atomic Oxygen Environmental Simulation Facility for Space Materials Evaluation," NASA TM-160128, 1993.

${ }^{20}$ National Institute of Standards and Technology Standard Reference Database \#78, NIST Atomic Spectra Database, Ver. 4, Nov. 2011.

${ }^{21}$ Rutledge, S.K., and Banks, B.A., "Techniques for Synergistic Atomic Oxygen and Vacuum Ultra Violet Radiation Durability Evaluation of Materials for Use in LEO," NASA TM-107230, 1996.

${ }^{22}$ Fridman, A., Plasma Chemistry, Cambridge Univeristy Press, New York, 2008.

${ }^{23}$ Caledonia, G.E., and Krech, R.H., "Energetic Oxygen Atom Material Degradation Studies," $25^{\text {th }}$ Aerospace Sciences Meeting, AIAA-87-0105.

${ }^{24}$ ASTM E 2089-00, “Ground Laboratory Atomic Oxygen Interaction Evaluation of Materials for Space Applications," ASTM International, West Conshohocken, PA. Reapproved in 2006.

${ }^{25}$ Seren Industrial Power Systems, Product Catalog, 2008. Vineland, NJ.

${ }^{26}$ Dassault Systemes SolidWorks Corporation, Solidworks, Software Package, Student Edition 2011, Waltham, MA. 
${ }^{27}$ Hamamatsu Corporation, Electron Tube Division, Light Sources: Deuterium Lamps, Jul. 2011, Iwata City, Shizuoka Pref., Japan.

${ }^{28}$ Tennyson, R.C., and Hughes, P.C., "Long Duration Exposure Facility Surface Temperatures," Journal of Spacecraft and Rockets, 1992. Vol. 29, No. 1 (p96-101). DOI: 10.2514/3.26319. 


\section{Appendix A. Vacuum Chamber Operation Procedures}

1. Ensure that all vacuum control panel toggles are switched to the off position.

2. Make sure all service panels are closed and secure.

3. Flip the $1203 \Phi$ VAC breaker to the "on" position.

4. Open the ball valve to the pressurized air line.

5. Check the pressurized air regulator and ensure that it reads between $70-75$ psi.

6. Turn on the "Main Power" on the vacuum control panel.

7. Turn on the Granville-Phillips 375 Vacuum Gauge Controller. Convectron gauge 3 (CG3) indicates chamber pressure in Torr.

8. Make sure all ports are closed, including the green nupro valve on the gas insertion line.

9. Turn on the Mechanical Pump on the vacuum control panel.

10. Turn on the Chamber Roughing on the vacuum control panel. Monitor the chamber pressure on the Granville-Phillips 375 Vacuum Gauge Controller.

11. To shutdown, close the Chamber Roughing valve and Mechanical Pump. Engage the vent valve, raise the hoist when pressure has equalized. 


\section{Appendix B. Atomic Oxygen Apparatus Operation Procedures}

\section{Part I. Internal Vacuum Assembly}

\section{Part Ia. Upper Assembly}

1. Insert the two 3/8"-24 rods into the endplate of the vacuum chamber. Place one nut on each rod approximately 1-2 inches up the shaft.

2. Assemble the RF electrode and Dark Space Shield (DSS) according to the drawing in Chapter IV, Figure 11.

2a. Check to make sure the surface of the aluminum electrode is clean; free of grease, oils, dirt, etc. Place the electrode face down on a soft, clean surface.

2b. Place the four alumina washers (1/4" ID by 0.070 " thick) over the four $1 / 4$ "-20 blind holes.

2c. Place the DSS on top of the RF electrode, aligning the 1/4" through holes on the DSS with the 1/4"-20 blind holes on the electrode.

2d. Connect the DSS and electrode with the 4 four $1 / 4$ "-20 by $11 / 2$ " while using the 1/4" ID by 1 " shoulder washers.

3. Mount the electrode/DSS assembly to the two 3/8"-24 rods inside the chamber. This will require 2 people.

3a. Place two washers, 3/8" ID washers on the top of the DSS

3b. Lift the electrode/DSS assembly with washers up onto the 3/8"-24 rods.

3c. Once the assembly is in place, secure the assembly with two $3 / 8$ "-24 nuts. Thread the nuts on until 1 or 2 threads on the threaded rods is showing.

3d. Lower the assembly down onto the lower 3/8"-24 nuts, tighten the upper $3 / 8$ "'-24 nuts to secure the upper assembly.

3e. Check to make sure the DSS is level, if necessary adjusted the height of the $3 / 8$ "-24 nuts.

4. Attached the 2" wide ground strap to the endplate of the vacuum chamber using the \#10 vented screws and the 3/8"-24 bolt. Use washers and necessary to ensure intimate contact. 


\section{Part Ib. Lower Assembly}

1. Assemble the cross support system by connecting the 2" wide aluminum cross.

2. Attach one of the two stainless steel L-brackets from the internal chamber mounts to the front of the aluminum cross assemble.

3. Attach 2" wide copper ground strap to the bottom of the ground plate using the \#10 vented screws that are 1/4" long. Use washers and necessary to achieve tight and intimate contact between the ground strap and ground plate. The copper ground strap should lie on the outside of the SS L-bracket. Loosen the \#10 vented screws to allow for horizontal adjustment of the copper grand strap.

3. Screw in the $1 / 4$ "-20 by 4 " rods into the ground plate. Place one nut approximately 1" above the ground plate on each rod.

4. Place the cross assembly above the ground plate and screw the $1 / 4$ "-20 rods to the grounding plate to secure the plate.

5. Take the assembly to the chamber and attach two type $\mathrm{K}$ thermocouples. One close to the center of the ground plate, and one close to the edge of the ground plate. Avoid all holes on the ground plate.

6. Secure the ground assembly by attaching the back end of the aluminum support cross to the rear SS L-bracket. Attached the front part of the assembly by using two \#10 screws, going through the SS L-bracket and copper strap, and attach the assemble to the chambers internal mounting plate.

7. Tighten the \#10 vented screws to secure the copper ground strap to the ground plate.

8. Insert the $1 / 4$ " SS tube into the center of the ground plate and the gas insertion feedthrough.

\section{Part Ic. Electrical assembly}

1. Connect the RG-393 coaxial cable to the electrical feedthrough by using a \#10 nut and bolt through the ring connectors.

2. Cover the ring connectors as best as possible with the small diameter Teflon PTFE tubing.

3. Cover the entire ring connector section with a longer, large diameter Teflon PTFE tube.

4. Place the alumina insert into the electrical slot on the dark space shield. 
5. Insert the female bullet connector into to the blind hole on the RF electrode. Make sure the connector is protected with Telfon PTFE tubing. If the female bullet connector is not tight and secure in the RF electrode. If the connector does not make a tolerance fit, remove the connector and enlarge the connector by spreading the slit.

6. Cover the lower electrical connection with a section of large diameter Teflon PTFE tubing.

7. Seal the large diameter Teflon PTFE tubing with Kapton tape, use 3-4 layers of tape.

8. On the vacuum electrical feedthrough, the copper feedthrough only has a thin Teflon PTFE coating. Cover this section with 3-4 layers of Kapton tape.

9. Cover the entire electrical cable with 3-4 layers of aluminum tape. The aluminum tape should be well grounded. At the vacuum electrical feedthough the tape should extend all the way up over the threads and nut and should make contact with the vacuum chamber endplate. At the DSS, the aluminum tape should extend from the cable to the dark space shield.

10. Cover all unused equipment with aluminum tape to prevent $\mathrm{AO}$ erosion. This includes unused thermal couples and electrical (BNC) feedhthoughs.

\section{Part II. External Assembly}

1. Move the power rack with the Seren R301MKII RF generator, the AT3 matching network, and the MC2 controller to the left side of the vacuum chamber.

2. Connect the RG-393 coaxial cable to the AT3 macthbox. The Type N connector should be hand tightened and secure, make sure the ring connector is pointed down towards the electrical vacuum feedthrough.

3. User a \#10 bolt and nut to connect the ring connectors on the RG-393 coaxial cable and the electrical vacuum feedthrough.

4. Attached the two zinc-coated flexible copper ground straps to the vacuum chamber endplate using the necessary washers and a \#8 screw.

5. Wrap all exposed electrical connectors with small and large diameter Teflon PTFE tubing. NOTE: make sure to cover the SS portion of the electrical vacuum connector, this is in contact with the copper center conductor and is therefore transmitting RF energy. 
6. Wrap the Teflon PTFE tubing with Kapton tape to eliminate any possible arc pathways. Use 3-4 layers.

7. Wrap the entire unshielded portion of the RG-393 coaxial cable with aluminum tape. The tape should eliminate all gaps in which RF can escape into the room. Ensure that the tape covers the entire feedthrough and is in contact with the vacuum chamber endplate; also ensure that the tape is in contact with the two flexible ground straps. Use 3-4 layers of aluminum tape.

8. Connect the two green 12-gauge ground cables to the electrical system. One should connector to the vacuum chamber endplate. The other should connector to the metal portion of the power rack frame, at the same connector with the two other green 12-gauge ground cables attached to the AT3 and R301.

\section{Part III. System checks}

1. Check to make sure the CTI cryotorr-10 cyropump is at room temperature, even if the gate valve is closed. If the cyropump is not a room temperature do not run the system. Running the AO system with the cryopump cold can lead to catastrophic failure of the entire chamber.

2. Check that the Welch vacuum pump has the proper oil level and oil type. While running the AO system the pump must have the Gold quality oil from Welch vacuum. Running the AO system without the proper oil can lead to catastrophic failure of the mechanical pump.

3. Check all ground paths for the RF energy.

3a. The R301 RF generator should be connected to the power rack shelf unit with a 2" copper strap.

3b. The AT3 matchbox should be connected to the power rack shelf unit with a 2" copper strap.

3c. Each copper strap from $3 \mathrm{a}$ and $3 \mathrm{~b}$ should be connected to the metal frame of the power rack using green 12-gauge wire with a \#10 nut and bolt.

3d. A green 12-gauge wire should connect the metal frame from the power rack to the vacuum chamber endplate. The same connection point from $3 \mathrm{c}$ should be used.

3e. The two zinc-plated flexible copper ground straps on the RG-393 coaxial cable should be connected to the vacuum chamber endplate.

3f. Inside the chamber, the DSS and the ground plate should be connected to the chamber walls with a 2" copper strap. 
3g. Inside the chamber, the RG-393 coaxial cable should be completely covered in aluminum tape. The tape should contact the DSS and the vacuum chamber endplate.

4. Check all electrical connections on the power supply system.

4a. Connect the power strip on the power to the outlet on the wall. The wall outlet should have a 20 A fuse. Tape over the second power outlet so it cannot be used.

4b. Connect the MC2 controller and the R301 generator to the power strip. Make sure the main power on the R301 is in the off position when plugging in. Tape over the rest of the outlets on the power strip so they cannot be used.

4c. Connect the MC2 and AT3 with the 25-pin silver coated analog cable. Secure the cable with the 25-pin connector screws.

4d. Connect the AT3 and the R301 with the 3 foot, RG-393 coaxial cable with the Type $\mathrm{N}$ connectors. The connectors should be handed tightened and secure.

5. Turn on the power strip.

6. Turn on the main power on the R301.

\section{Part IV. Sample preparation}

Use gloves at all times when handling the samples or the sample containment cover plate. Clean cover plate with IPA and chem-wipes if necessary.

1. Cut samples of Kapton HN into 2"x2" squares.

2. Outgas all samples in accordance to ASTM E2089: a minimum of 48 hours below 200 mTorr.

3. Remove samples from outgassing chamber and weigh in accordance to ASTM E2089: within 5 minutes.

4. Place the samples onto the ground plate over the 4 sample cell areas. The samples should fit between the eight 1/4"-20 screw holes.

5. Cover the samples with the sample containment plate. This is an arrow on the top surface of the ground plate and the bottom surface of the sample containment plate for proper alignment. 
6. Secure the sample containment plate with the eight low profile 1/4"-20 screws.

The short screw is used on the forward most hole, otherwise there will be interference with the 2 " ground strap on the ground plate.

\section{Part V. Operating procedures.}

1. Pump down the chamber as described in Appendix A.

2. Open the green nupro valve on the gas insertion line. Use the needle valve to adjust the pressure in the chamber to $175+/-10$ mTorr.

3. Turn on the R301 generator.

4. Set the power to 125 Watts.

5. Turn on the MC2 controller.

6. Adjust the load and tune capacitors to 50\%. Make sure the operational mode is in Automatic for both load and tune.

7. Turn on the RF power using the switch on the R301.

The capacitors on the MC2 should auto adjust and find a stable operational point where the reflected power is 0 watts. If there is still reflected power or if the capacitors motors begin to oscillate, turn off the system and refer to the MC2 manual.

8. Once a stable point has been achieve, adjust the phase and magnitude potentiometers on the left hand side of the AT3 until the output on the MC2 controller is $0+/-25 \mathrm{mV}$.

9. Maintain a pressure between 165-185 mTorr and record temperature values every hour.

\section{Part VI. Shut down procedures.}

1. Turn of the RF power switch on the R301 generator.

2. Close the green nupro valve on the gas insertion line.

3. Turn off the R301 and MC2 controllers.

4. Disconnect the Type N connector on the AT3 macthbox. 
5. Disconnect the two green 12-gauge ground cables from the power rack and the vacuum chamber endplate. This will allow for free movement of the vacuum chamber hoist and the power track.

6. Let the samples sit in the chamber under vacuum until the average temperature on the ground plate is under $35^{\circ} \mathrm{C}$.

7. Vent the chamber in accordance to Appendix A.

8. Remove the sample containment cover plate, and weigh the samples in accordance with ASTM E2089: within 5 minutes. Caution: the DSS will be hotter than the ground plate, avoid contact with the DSS.

9. Disassemble and store the apparatus as necessary. 


\section{Appendix C. Vacuum Ultraviolet Lamp Operation Procedures}

\section{Part I. Installation}

1. Install the lamp housing assembling on top of the 5" ConFlat 275 nipple.

2. Position the lamp inside the chamber to be $10.7 \mathrm{~cm}$ from the forward most sample cell.

3. Connect the lamp power supply to the DC power converter. Do not plug the converter in at this time.

3. Once the bell jar is in place and the hoist is lowered, connect the clear air hose to the facilities pressurized air line using the 1/4" Swagelok fitting. Connect the black power cable from the lamp housing to the cable on the lamp power supply.

\section{Part II. Operating procedures}

1. Open the hoke valve on the compressed air line, regulate the compressed air to 20 psi. Air should be continuously venting out of the top of the lamp housing.

2. Plug in the DC convert to the power rack power strip to turn on the lamp. The arc will take about 20 seconds to warm up and ignite.

\section{Part III. Shut down procedures}

1. Unplug the DC converter to turn off the lamp.

2. If running the AO plasma system, leave the compressed air running to keep the lamp cool. Turn off the compressed air at the hoke valve after Step 6 of Appendix B, Part VI.

3. If running the lamp independently, turn off the compressed air at the hoke valve.

4. Disconnect the compressed air line at the $1 / 4$ " Swagelok fitting. Place the hose on top of the vacuum chamber endplate.

5. Disconnect the black power cable. Place the cable on top of the vacuum chamber endplate.

It is now clear to vent the chamber and raise the hoist.

6. Recorded the total run time of the lamp in the log book.

7. Every 100 hours clean the Magnesium Fluoride lamp window per instruction in Reference 14, or per manufacturer's instructions. 


\section{Appendix D. Lessons Learned}

There are many nuances and peculiarities associated with this system that were discovered through trial and error or discussion with industry experts. This section is intended to document these issues and inform the reader of how these issues were corrected or mitigated, with the goal of bequeathing the knowledge gained during the design, manufacturing, and assembly of this apparatus.

Many issues were encountered with the RF electrical system. Grounding of the system is very non-intuitive. RF power is extremely dependent on the skin effect, meaning that all the current travels on a very thin outer layer of the conductor. The manufacturer recommended using silver plated copper straps; the conductors inside the power supplies are made in this manner. These could not be obtained in a reasonable time, so 2" copper straps were used when possible. It is important to keep the copper untarnished, as any surface discontinuities will not transmit RF energy. As continuous maintenance, the copper straps should be cleaned with vinegar regularly to keep and clean, shinny surface. For ease of cleaning, use a fine grit sandpaper (such as 220 or 500 grit) and vinegar to sand the surface of the copper. If any component is not grounded, the component will absorb and transmit RF energy, and as far as the electrical system is concerned said component is now an inductor. This will greatly increase the load of the system, and the matching network will not be able to find a stable operation point.

The skin effect also dictates which type of electrical connectors must be used. The largest possible surface area is desired, as this will allow for the path of least resistance. At the same time, it is not advantageous to make the RF energy travel farther than necessary. For example, don't use long bolts on the ring connectors, as the RF will have 
to transmit to the end of the bolt and back, causing a reflected signal and lost power. These connectors also need to be robust; the original design used male and female bullet connectors. These connectors would melt during high power or long duration testing. The solder joints on the ring connectors will also melt during operation. As part of regular maintenance, disassemble the electrical system and check to the solder to see if it melted and flowed away. Re-solder or replace connections as necessary. The system has now been designed to prevent this from happening, but it is still a young system and needs to be regularly inspected.

With regard to finding a matched operation point that minimizes the reflected power and forms a stable plasma, the manuals will outline how to adjust the AT3 matchbox to change the operational range. This is necessary when the controller experiences chattering, meaning it reaches the operational limit (either upper or lower) and begins to oscillate in an attempt to find a match point. Before taking apart the system try to manually adjust the system first. Occasionally the automatic setting will try to match to a non-ideal match point. There are multiple possible operational points depending on the primary load path of the plasma. We want the plasma to generate between the two plates; however, the automatic matching will sometimes try to obtain a matched point on the load path from the RF electrode and the chamber wall. Simply place the MC2 in manual mode and adjust the variable capacitors until the primary plasma is between the plates and the reflected power is now zero Watts. I recommend using a structured, grid method. Adjust one capacitor in intervals of 5\% will traversing the other capacitor all the way up and down. Try to find a range where the maximum power is forwarded and the minimum power is reflected; once this region is discovered try fine 
turning to find the match point. To make this process easier, increase the pressure in the chamber by inserting more air, 200 mTorr should suffice and do not surpass 400 mTorr. However, it is incredibly bad and dangerous to run the system with reflected power for any duration greater than a few minutes. If necessary, perform diagnostics over multiple intervals, allowing the power supply and matchbox to sit idle with the power off for 1520 minutes.

The final recommendations are in regard to sample handling and contamination mitigation. It can be difficult to weigh the samples within 5 minutes of removal from the outgassing chamber. Two people make the process easier and faster. Wear gloves at all times and prevent any oils, grease, or contaminants from touching the sample. The scale can sometime have anomalous readings, so it is good practice to weigh the samples multiple times to find an average value. Also make sure to calibrate and level the scale per the manufactures instructions. Finally, try to avoid any excess grease, adhesives (such as tape backing), or volatile substances in the chamber. Most greases and adhesives are silicon based and will react with the AO and contaminate samples. Tape is a necessary evil; try to prevent any adhesive from being exposed to the plasma, only aluminum should be exposed. Cover all Kapton tape with aluminum tape, otherwise it will erode away and become useless.

If at any time there is any uncertainty or doubt with the setup stop, recheck all systems, and check with another technician to confirm or alter the setup. Incorrect use can cause damage or harm to the apparatus, power supplies, and users. Safety first. 